\title{
A Survey on Gait Recognition via Wearable Sensors
}

\author{
MARIA DE MARSICO and ALESSIO MECCA, Sapienza University of Rome, Italy
}

\begin{abstract}
Gait is a biometric trait that can allow user authentication, though it is classified as a "soft" one due to a certain lack in permanence and to sensibility to specific conditions. The earliest research relies on computer vision, especially applied in video surveillance. More recently, the spread of wearable sensors, especially those embedded in mobile devices, has spurred a different research line. In fact, they are able to capture the dynamics of the walking pattern through simpler one-dimensional signals. This capture modality can avoid some problems related to computer vision-based techniques but suffers from specific limitations. Related research is still in a less advanced phase with respect to other biometric traits. However, many factors the promising results achieved so far, the increasing accuracy of sensors, the ubiquitous presence of mobile devices, and the low cost of related techniques - contribute to making this biometrics attractive and suggest continuing investigating. This survey provides interested readers with a reasoned and systematic overview of problems, approaches, and available benchmarks.
\end{abstract}

CCS Concepts: • General and reference $\rightarrow$ Surveys and overviews; • Security and privacy $\rightarrow$ Authentication; Biometrics; $\bullet$ Human-centered computing $\rightarrow$ Ubiquitous and mobile computing systems and tools; Smartphones; Mobile devices; $\bullet$ Computer systems organization $\rightarrow$ Embedded hardware;

Additional Key Words and Phrases: Biometrics, gait recognition, mobile devices, embedded sensors

\section{ACM Reference format:}

Maria De Marsico and Alessio Mecca. 2019. A Survey on Gait Recognition via Wearable Sensors. ACM Comput. Surv. 52, 4, Article 86 (August 2019), 39 pages.

https://doi.org/10.1145/3340293

\section{INTRODUCTION}

Modern technologies facilitate everyday life but also raise unprecedented security issues. Robust user authentication is needed to prevent violation of restricted physical areas (e.g., a bank caveau) but also of remote services (e.g., home banking), or mobile devices (e.g., smartphones/tablets). Authentication processes are conventionally grouped into three classes: knowledge-based, object (or token)-based, and the most recent biometrics-based [10]. The first one relies on a secret the user has to know/remember, e.g., passwords and PIN codes. Studies on users' habits in managing passwords $[27,37,43]$ have pointed out memorability problems, especially for robust enough passwords. The consequent tendency to reuse them over different services raises a security breach in itself. The second class is characterized by possession, e.g., of physical keys. These two approaches can be combined (e.g., a bank-card with PIN code). Biometric authentication rather exploits something

Work supported by Grant PRIN 2015 COSMOS: "COntactlesS Multibiometric mObile System in the wild" funded by the "Italian Ministry of Education, University and Research," Grant No. 201548C5NT.

Authors' addresses: M. De Marsico and A. Mecca, Sapienza University of Rome, Via Salaria, 113, Rome, 00198, Italy; emails: \{demarsico, mecca\}@di.uniroma1.it.

Permission to make digital or hard copies of all or part of this work for personal or classroom use is granted without fee provided that copies are not made or distributed for profit or commercial advantage and that copies bear this notice and the full citation on the first page. Copyrights for components of this work owned by others than ACM must be honored. Abstracting with credit is permitted. To copy otherwise, or republish, to post on servers or to redistribute to lists, requires prior specific permission and/or a fee. Request permissions from permissions@acm.org.

(C) 2019 Association for Computing Machinery.

0360-0300/2019/08-ART86 \$15.00

https://doi.org/10.1145/3340293 
a person is, i.e., either physical or behavioral characteristics. Various human traits can be used as biometrics, e.g., the popular fingerprints, the face, the iris, the hand geometry, the gait, and so on. These features must obey specific conditions, especially universality (the trait must be owned by all subjects), and permanence (the trait must remain stable for sufficient time). Traits difficult to spoof (maliciously reproduce) are preferred. In this sense, behavioral ones, e.g., gait or signature dynamics, are interesting candidates, as they are more difficult to imitate. However, they may lack permanence, being possibly conditioned by physical and/or emotional factors. In fact, biometric traits are classified as hard and soft. Hard ones generally allow a sufficiently accurate subject recognition, though suffering from trait-specific problems. For instance, face recognition can be hindered by pose, illumination, and expression (PIE) variations. Soft traits either identify entire groups of individuals (e.g., the hair color) or lack sufficient permanence (e.g., most behavioral traits can be affected by emotional state). Gait is considered a soft trait, but several studies are still investigating its discriminative power. Gait recognition entails computer vision techniques, processing video data, or the analysis of signals from either equipped floors or wearable sensors.

Mobile biometrics play an increasing role in different scenarios, allowing ubiquitous applications at relatively low costs, and avoiding further capture equipment. This survey proposes a comprehensive discussion of gait recognition by wearables. The different sensors embedded in everyday mobile devices, especially smartphones, make this approach more and more affordable. The reverse of the medal is a diffusing concern for a possible privacy violation. These two aspects make the subject presently relevant from both a social and a scientific perspective. ${ }^{1}$

Compared to the recent survey in Reference [88], this article reviews the most recent literature, and also provides more details on theoretical aspects and problems of gait recognition and a more coordinated overview of the single approaches. Section 2 introduces basics on biometric systems and general gait recognition concepts; Section 3 focuses on wearable sensors used in gait recognition; Section 4 describes the publicly available datasets; Section 5 reviews popular preprocessing techniques used to improve the signal quality; Section 6 presents the literature on recognition, classifies the approaches according to processing choices, and sketches a schematic overview; Section 7 lists some works addressing gait recognition in less controlled settings; Section 8 deals with some more specific issues; Section 9 reviews some works investigating possible spoofing in wearable sensor-based gait recognition; Section 10 provides some more discussion and closes the article.

\section{INTRODUCTION TO GAIT BIOMETRICS}

Biometric traits can be physical or behavioral and can be further categorized into hard and soft ones. The former traits present properties of universality, uniqueness (discriminative power), permanence (in the long term), and ubiquitousness, that allow accurate recognition, especially in controlled conditions. The latter lack in one or more of such properties. Soft physical traits, e.g., hair color, height, or age, do not identify a single subject but a group. However, they can be useful to reduce the recognition space. Most behavioral traits fall in the soft category too, due to the lack of sufficient permanence. For instance, the way of walking, writing/signing, or the keystroke dynamics may be affected by either physical or emotional status, especially over the long term. Nevertheless, they can be used for continuous reidentification, e.g., to assure that the same single person works with a keyboard during a session. Being not bound to physical appearance but to the subject's "way to be or behave," they are more difficult to forge and spoof, and can also be

\footnotetext{
${ }^{1}$ Online identification is getting more and more intrusive-phones can now tell who is carrying them from their users' gaits. https://www.economist.com/science-and-technology/2019/05/23/online-identification-is-getting-more-andmore-intrusive.
} 
used together with hard ones, to improve recognition accuracy. Among soft biometrics, gait, i.e., the recognition of people by the way they walk, is increasing its popularity in the last years due to new capture possibilities.

In a biometric system, a set of known subjects is enrolled with one or more biometric templates (gallery). An incoming template (probe) is matched by similarity/distance against the gallery, by three kinds of recognition operation.

Verification: $1: 1$ identity matching between the probe and the gallery templates of the claimed identity (e.g., implicitly, being the owner of a smartphone). A threshold is set up to decide the acceptance/rejection of the claim. False Acceptance Rate (FAR; also False Match Rate-FMR), and False Rejection Rate (FRR; also False Non-Match Rate-FNMR), estimate system accuracy at different thresholds, with respective curves. FAR and FMR (FNMR and FRR) are often used interchangeably in the literature. Their difference is that FMR and FNMR refer to the accuracy of the recognition algorithm, while FAR and FRR are system-level errors which include the failure to acquire or compare a certain number of samples. Equal Error Rate (EER, the intersection of these two curves) is used as a summative measure. In addition, Receiver Operating Characteristic (ROC) plots Genuine Acceptance Rate $(\mathrm{GAR}=1-\mathrm{FRR})$ vs. FAR, and its Area Under Curve (AUC) can also be considered.

Identification Closed Set: no identity claim, 1:N matching of the probe with all gallery templates, assuming that it surely belongs to an enrolled user. The returned identity is the first in the gallery ordered by distance/similarity with the probe. The rate of correct recognition in the first position (Recognition Rate-RR) is the main performance measure. A more detailed accuracy evaluation is given by plotting as Cumulative Match Curve (CMC) the achieved Cumulative Match Score (CMS) values for each rank $k$ (rate of correct identification within the first $k$ positions).

Identification Open Set: 1:N matching, no assumption on the presence of the probe identity in the gallery. Acceptance is regulated by a threshold allowing a Reject option. The probe has to be "close enough" to the first result in the returned ordered list, to meet the threshold value, but also the first result must correspond to the correct identity. Therefore, verification is used in more critical settings (e.g., access control to secure places), but Identification Open Set is the recognition operation raising more errors. Though being definitely more realistic than Identification Closed Set, it is rarely addressed in the literature. The Detection and Identification Rate at rank $k$ for threshold $t-\operatorname{DIR}(k, t)$ is the percentage of genuine tests in which the probe meets the threshold and the correct identity is returned within the first $k$ positions. FAR is computed as in verification while FRR is defined as $1-\operatorname{DIR}(1, t)$, encompassing both the case of a genuine probe not meeting the threshold and the case when the correct identity is not in the first place. The evaluation relies again on EER, ROC, AUC.

\subsection{General Gait Physiology and Individual Characteristics}

The human gait is produced according to strict bio-physiological rules. According to medical literature [94], the locomotor planning starts in the brain. As for any kind of movement, it is necessary to convert the "idea" of the desired movement into the pattern of muscle activity necessary for walking [24]. The next step entails the transmission of the neural output. The final implementation of the command can be divided into task planning and plan execution. The muscle activation produces tension, that in turn generates the joints movement. The joints forces and moments cause the rigid skeletal links (segments such as the thigh, calf, foot, etc.) to move and to exert forces on the external environment. The gait action itself triggers the generation of ground reaction forces, applied through the feet. Such reaction forces support the body and avoid its displacement during walking. The act of walking requires both the periodic movement of each foot from one position of support to the next and sufficient ground reaction forces, no matter how distorted the pattern may 


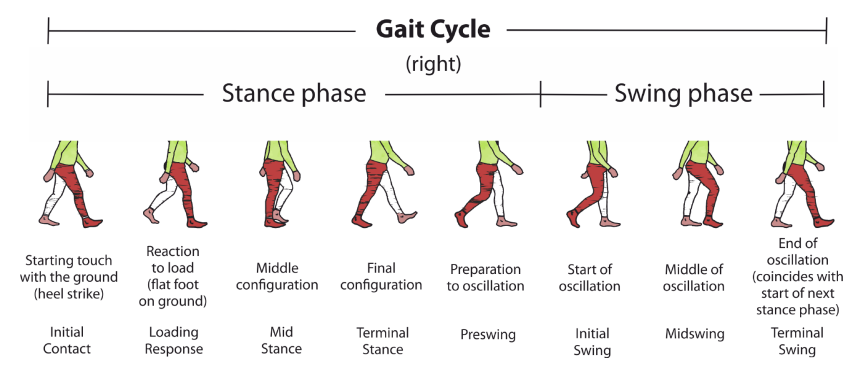

Fig. 1. Walk cycle dynamics (inspired by Reference [94]).

be by underlying pathology [83]. The periodic leg movement is the essence of the cyclic nature of human gait. Each gait period can be divided into 2 phases and 8 configurations [94], as in Figure 1.

The entire gait period is generally called cycle, while the first four configurations and the next four ones compose the right step and the left step (or vice versa). Women generally present shorter steps and more pelvic movements, while intra-subject differences can be caused by aging.

Given the above aspects, it is worth wondering at which extent this trait can be considered distinctive of different subjects. As underlined in Reference [8], basic walking characteristics are naturally stereotyped, and basic kinematic patterns are invariant across the normal range of speeds: locomotion must preserve postural stability and dynamic equilibrium [65]; possible changes in support conditions call for an anticipatory adaptation involving coordinated synergies of upper limb, trunk and lower limb movements [23]; the head is normally stabilized in space both during posture and locomotion, and allows to monitor gravity direction [79]. However, different personal energy-saving strategies produce qualitative and quantitative features, making individual walking styles unmistakably recognizable. Experiments in Reference [76] by electromyographic (EMG) signals show that locomotion cannot be considered as a completely stereotyped function since, "despite the similar kinematics, the torque time courses of different subjects present significant differences in agreement with different temporal sequence of muscle activation." A later work [77] investigates sequences of muscle forces providing for coordinated gait. The individual posture and locomotion have long been defined as the signature traced by the body [3]. The individual characteristics of gait are investigated in Reference [6] with the aim of identifying kinematic principles that may partially explain this behavioral variance. Different kinematic strategies are among the factors that produce inter-personal differences in walking patterns, and that can, therefore, be exploited for identification.

\subsection{Approaches to Gait Recognition}

The gait dynamics shortly described in Section 2.1 (see References $[6,94]$ and other works mentioned in the section) produce visual patterns, dynamic weight/pressure distributions, and acceleration patterns on the 3D space axes that are characteristic and that can discriminate between different users. Accordingly, the approaches in the literature to gait recognition fall into three main groups [30].

Machine Vision-based: They model static/dynamic features of walking through visual features.

Floor Sensors-based: They exploit floors equipped with special sensors, e.g., for pressure and weight, capturing properties more related to quantitative physical user's features than to appearance.

Wearable Sensors-based: They use sensors directly worn by the user, and capture pure walking dynamics; there is no need to equip the environment, allowing ubiquitous recognition. 
As generally happens for the other biometric traits, gait recognition is affected by both interpersonal similarities and intra-personal differences. Variations in the gait pattern can depend on, for example, walking speed, kind of worn shoes, ground slope, and ultimately on some temporary illness. If the source of data is a camera, then gait recognition can be further affected by different kinds of worn dresses and by carried objects [99]. In this case, these factors add to the problems generally negatively affecting image processing, e.g., varying illumination, occlusion, pose, and different camera view. Floor sensor-based gait recognition has been abandoned during recent years because of the low recognition accuracy achieved and the cost of acquisition devices. However, the biomedical field $[50,51]$ still exploits gait analysis by equipped-floors. Wearable sensors appear as a promising alternative to reduce the recognition problems related to appearance.

Notwithstanding limitations, gait recognition presents some good aspects too: (1) it can operate at variable distances (in Machine Vision approaches, the distance is about 10 meters, while in the cases of Floor Sensor and Wearable Sensor approaches the acquisition devices are either inside the floor or located on the user body); (2) it is non-intrusive, since it does not require any specific user action but walk; (3) it is non-invasive, since it generally does not require any physical contact with an acquisition device; (4) it is quite difficult to copy or forge; (5) though it is a soft biometrics, recent works report interesting results in terms of recognition accuracy, and it can be combined with other "hard" biometrics for better recognition and/or anti-spoofing.

Since a complete review of gait recognition is out of the scope of this article, in the following only wearable sensor-based proposals are surveyed. Interested readers can find reviews of machine vision-based techniques in References [53, 80, 82] and three proposals about floor sensor-based recognition in References [62, 73, 89]. The latter especially point out how gait analysis [20] can also be exploited for diagnostic/clinical aims (interested readers can refer to Reference [64]). Finally, gait often appears in researches dealing with human action recognition; extensive reviews can be found in References [4, 9, 78, 93].

\section{WEARABLE SENSORS FOR GAIT RECOGNITION}

"The growing popularity of wearable devices is leading to new ways to interact with the environment, with other smart devices, and with other people. Wearables equipped with an array of sensors are able to capture the owner's physiological and behavioral traits, thus are well suited for biometric authentication to control other devices or access digital services." This quotation from the survey presented in Reference [7] points out a new trend in biometric research, focusing on cheap, widely available and easy-to-set-up wearable equipment. Such equipment, and in particular smartphones where they are embedded, can further allow a non-intrusive autonomous sensing and user context recognition, spurring a new class of applications, defined as opportunistic user context recognition with mobile phones, which are the topic of another recent survey [44] and of a number of recent works [5, 14, 18, 19, 57].

As already mentioned, the gait produces a pattern of accelerations over the three axes in the 3D space. Such accelerations can be captured by sensors integral with the body parts, especially those involved in the movement (wearable sensors). The produced pattern is characteristic of each individual. Figure 2 shows three signals captured over the y-axis (in general the most informative one): two signals are from the same subject (the reddish/lighter ones), while one is from a different subject (the darker one). The abscissa reports the temporal order of the samples. It is possible to appreciate both the intra-subject similarities and the inter-subject differences. Analogous considerations hold for other physical quantities, captured by the wearable sensors shortly described in the following (see also Reference [64]).

Accelerometers are the presently most used wearable inertial sensors for gait recognition, either embedded in other devices or not. They measure the proper acceleration, also known as 


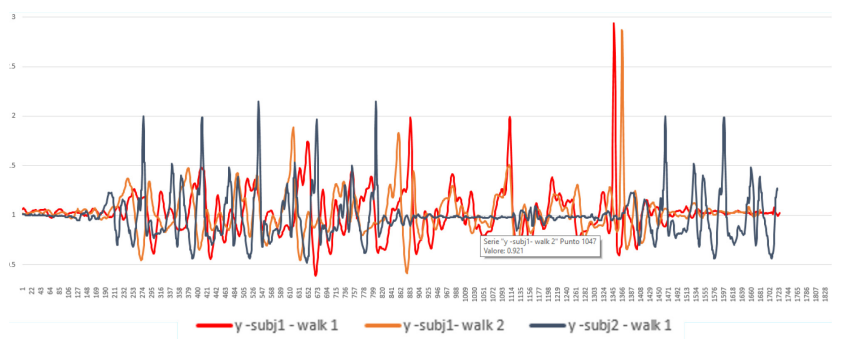

Fig. 2. Two signals from the same subject and one from a different subject. All signals are captured on y axis.

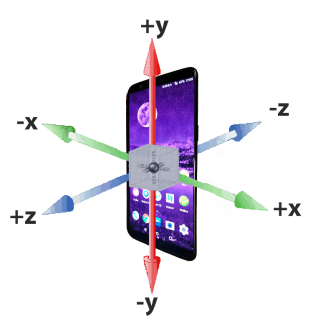

(a)

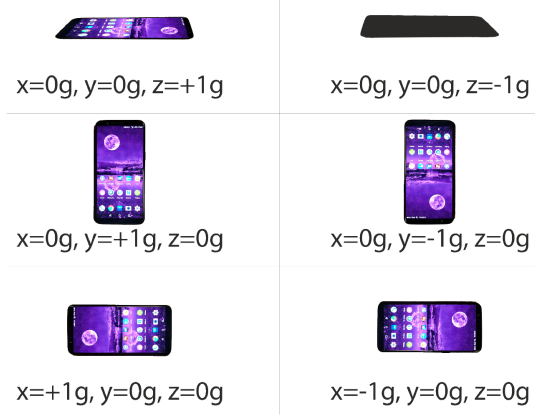

(b)

Fig. 3. (a) Axes and their orientations of the accelerometer in smartphones. (b) Ideal accelerometer values.

$\mathrm{g}$-force, which is the one relative to freefall. This is the acceleration experienced by people and objects. From a conceptual point of view, an accelerometer acts as a mass hung up by three springs. When it is subject to an acceleration, the mass moves from the static position consistently with the casing, modifying the length of the springs. The acceleration value is then computed measuring the amount of displacement with respect to the static position, in the three directions. Data at a given time consists of the triplet of acceleration values registered for the $x, y$, and $z$ axes. Like most sensors, accelerometers are subject to calibration problems. In fact, even the measures provided by two accelerometers from the same model and brand in identical conditions are slightly (or even very) different from each other. This can be easily verified by putting two smartphones on a plain surface and running any application showing the accelerometer values. Among the main accelerometer characteristics, an important property is linearity, i.e., its response is directly proportional to the physical acceleration to measure. This can be exploited in various ways, e.g., for data normalization, better discussed in Section 7. Bandwidth is another important parameter (often referred to as sampling rate), i.e., the maximum frequency of detectable variations, measured in $H z(1 / s)$. Further characteristics are not relevant for gait recognition. Figure 3(a) shows the position and the orientation of the accelerometer axes in a smartphone. Figure 3(b) reports the ideal values of acceleration over the three axes in all of the six "flat" positions, when the sensor is embedded in mobile devices.

Gyroscopes are another kind of inertial sensors, measuring a triplet of rotation angles along three axes. The results of joint use with accelerometers are controversial.

Accelerometers and gyroscopes are the most used sensors for gait recognition, because they are embedded in all recent smartphones. In modern devices, they are generally integrated into a single 


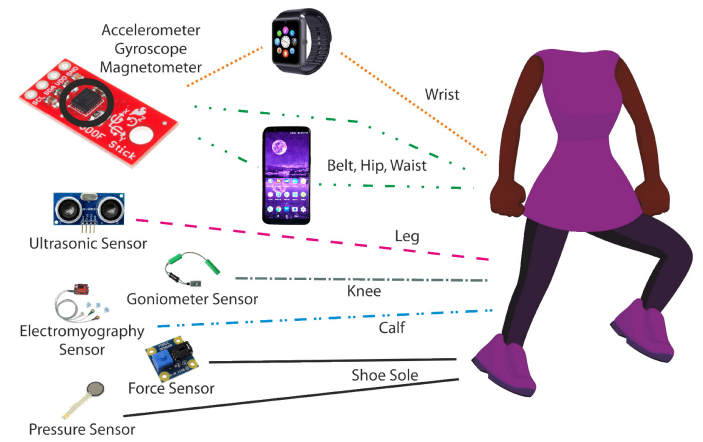

Fig. 4. Different wearable sensors and where they are usually worn (lines with different color/pattern).

component of micro electro-mechanical systems (MEMS) type, which sometimes includes also a magnetometer. This latter sensor measures the magnetic field. It is not used in gait recognition, because it can at most be exploited to determine the direction of the walk. This sensor is generally used for the geolocalization instead.

Further sensors are used in gait analysis, though some of them are not or not yet exploited in gait recognition too. They can be part of wearable devices or implanted inside a shoe toe or sole.

Force sensors stem from floor-based approaches. They measure the Ground Reaction Force (GRF) under the foot and return a current or voltage proportional to the pressure measured.

Pressure sensors rather measure the force applied to the sensor without taking into account its spatial components. They can be capacitive, resistive piezoelectric and piezoresistive sensors. The choice depends, e.g., on the range of pressure it is expected to stand and measure, and sensitivity. These sensors have been recently used for gait recognition too (see Section 8.4).

Goniometers measure the angles for ankles, knees, hips, and metatarsals. Strain gauge-based goniometers work with resistance that changes with sensor flexion (the material stretches, and the current traversing it has to travel a longer path, causing a proportional resistance increase). These sensors can be fitted in instrumented shoes but have never been exploited for gait recognition.

Ultrasonic sensors are used to analyze short steps and stride length, and feet distance.

Electromyograph (EMG) measures the electrical manifestation of either voluntary or involuntary muscle contraction. The signals can be obtained either non-invasively with surface electrodes, or invasively with wire/needle electrodes. They can measure different gait features, e.g., kinematic plots of joint angular motion; a recent work uses this technique (see Section 8.4).

Figure 4 sketches the possible positioning of the various wearable sensors on different body parts (distinguished by color and pattern of lines in the figure).

\section{GAIT DATASETS ACQUIRED BY WEARABLE SENSORS}

It is difficult to sketch a meaningful comparison of works in literature, because there are few public benchmarks. Furthermore, they are often neglected, contrary to, e.g., face recognition research. Most works use in-house data, and the former experiments mainly deal with special-purpose sensors. Those collections will be described with the works exploiting them, and their characteristics will be summarized at the end of Section 6. This section rather describes the only three (to the best of our knowledge) public freely accessible datasets relevant for this field. Table 1 summarizes their main features. Figure 5 allows appreciating both the differences in the signal shape across axes from the same device and across devices. On the abscissa, the temporal ordering of samples. 
Table 1. Summary of Freely Available Datasets that Were Collected by Wearable Sensors

\begin{tabular}{|c|c|c|c|c|c|c|c|c|c|}
\hline \multicolumn{3}{|c|}{ Data } & \multicolumn{7}{|c|}{ Dataset } \\
\hline Paper & Year & Dataset & Device(s) & $\begin{array}{c}\text { Device(s) } \\
\text { Position(s) }\end{array}$ & \# Subjects & Walk Length & $\begin{array}{l}\text { \# Walks per } \\
\text { Subject }\end{array}$ & \# Sessions & Notes \\
\hline [66] & 2014 & $\begin{array}{l}\text { OU-ISIR Inertial } \\
\text { Sensor }\end{array}$ & $\begin{array}{c}\text { 3 IMUZ, } 1 \\
\text { Motorola ME860 } \\
\text { (both with } \\
\text { accelerometer } \\
\text { and gyroscope) }\end{array}$ & Hip, Back Waist & 744 & $\begin{array}{l}15 \mathrm{~m} \text { (2 slopes } \\
\text { of } 3 \mathrm{~m}, 1 \text { level } \\
\text { of } 9 \mathrm{~m} \text { divided } \\
\text { into } 2 \text { of } 4.5 \mathrm{~m})\end{array}$ & $\begin{array}{l}1 \text { (manually } \\
\text { segmented } \\
\text { into } 4 \\
\text { fragments) }\end{array}$ & 1 & $\begin{array}{l}\text { Age ranges } \\
\text { from } 2 \text { to } \\
84 \text { years Short } \\
\text { signals }\end{array}$ \\
\hline [101] & 2015 & ZJU-gaitacc & 5 Wii Remote & $\begin{array}{l}\text { Left Upper Arm, } \\
\text { Right Wrist, } \\
\text { Right Hip, Left } \\
\text { Thigh, Right } \\
\text { Ankle }\end{array}$ & $\begin{array}{l}153(2 \text { sessions }) \\
+22(1 \text { session })\end{array}$ & $20 \mathrm{~m}$ & 12 & $\begin{array}{c}2 \text { (with } \\
6 \text { walks per } \\
\text { user each) }\end{array}$ & $\begin{array}{c}\text { Data are } \\
\text { interpolated }\end{array}$ \\
\hline [13] & 2016 & $\begin{array}{c}\text { BWR- } \\
\text { MultiDevice }\end{array}$ & $\begin{array}{c}1 \text { Samsung S4 } \\
\text { Active, } 1 \\
\text { OnePlus One, } 1 \\
\text { Sony Xperia S }\end{array}$ & Hip & 25 & 10 steps & $\begin{array}{c}12 \text { ( } 4 \text { for } \\
\text { each smart- } \\
\text { phone) }\end{array}$ & $\begin{array}{l}2 \text { ( } 2 \text { walks per } \\
\text { smartphone } \\
\text { per user) }\end{array}$ & $\begin{array}{c}3 \text { different } \\
\text { acquisition } \\
\text { devices }\end{array}$ \\
\hline
\end{tabular}

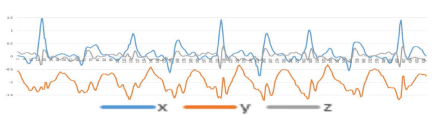

(a) $O U-I S I R$.

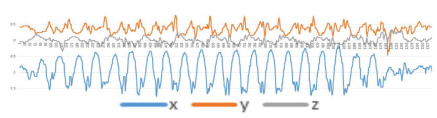

(b) ZJU-gaitacc.

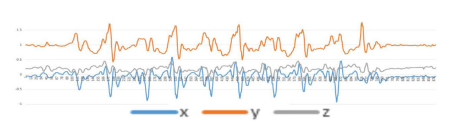

(c) BWRMultiDevice.

Fig. 5. Examples of acceleration signal from the three mentioned datasets. Differences in the signal shape can be appreciated both across axes from the same device and across devices.

OU-ISIR ${ }^{2}$ [66] collects data from a high number of subjects (744) with an age range of 2-78 years. No further demographic information is provided. The data come from both accelerometer and gyroscope, tough collected from a similar body location (a belt is located in the hip and back waist zones). A single signal per subject is collected during a single walk (very few intra-class variations) along a path with two slopes $3 \mathrm{~m}$ long (one ascending and one descending), and a level floor of $9 \mathrm{~m}$ ( $4.5 \mathrm{~m}$ in each direction). The diversity of ground inclination is a positive feature. The acceleration is collected using three IMUZ (with built-in accelerometer and gyroscope) and a smartphone (Motorola ME860 with built-in triaxial KXTF9 Kionix accelerometer). For each walk, it is possible to have access to seven signals (three from the accelerometer, three from the gyroscope of IMUZ, and one accelerometer signal from the smartphone). The total number of acceleration samples per walk is about 1,400 and no data preprocessing is applied. Each collected walk is manually segmented so that four fragments are extracted out from it, two from the level floor path and two from ground slopes, which are probably too short to provide a reliable benchmark. In fact, there are about 400 samples for the level floor and about 250 for the slopes, which seriously hinders recognition.

ZJU-gaitacc dataset ${ }^{3}$ [101] collects data from a lower number of subjects, but walks are much longer and varied. It includes the signals from 175 subjects, with 153 out of these participating in two different sessions, with a delay ranging from a week to six months, and with a high number of walks per subject (six per session). No individual demographic information on either gender or age is provided. In each session, the subject is asked to walk six times along a $20 \mathrm{~m}$ hallway. This corresponds to signals with a sufficiently high number of samples per walk (about 1,400). The acceleration data are collected using five Wii Remote controllers, located in the left upper arm, right wrist, right hip, left thigh, and right ankle. The positive feature of this dataset is the

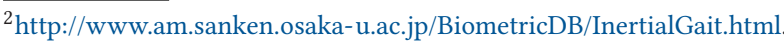

${ }^{3}$ http://www.cs.zju.edu.cn/ gpan/database/gaitacc.html.
} 


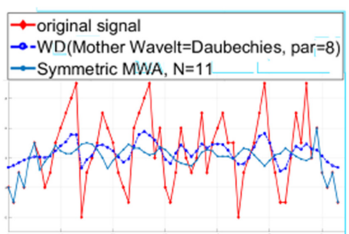

(a) The effect of wavelet denoising and moving weighted average.

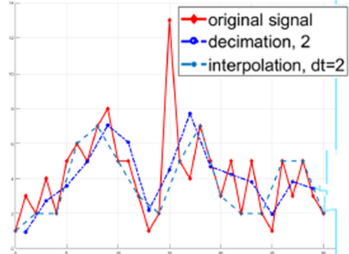

(b) Interpolation and decimation $(d t=2)$.

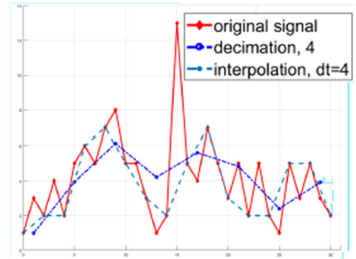

(c) Interpolation and decimation $(d t=4)$.

Fig. 6. Examples of denoising, interpolation, and decimation; the latter are on the same original signal.

high number of walks per subject and per sensor, and the signals have a sufficient length for a significant matching. Having two different sessions sufficiently separated in time allows also to consider more unpredictable time-related variations. However, the quality of data recording is quite low, due to the low accuracy of the controller. In addition, the data are interpolated, and, at the best of our knowledge, access to the raw data is not possible. This may be negative for approaches not entailing interpolation.

Concluding, OU-ISIR can assess inter-class variations, while intra-class ones are better captured by ZJU-gaitacc. The lack of demographic data impedes, e.g., to study gait-based gender recognition.

BWR-MultiDevice ${ }^{4}$ dataset exclusively collects data for smartphone-based experiments [13]. It is smaller than the previous ones but has the aim to highlight the differences among signals collected by different devices. It contains walking signals from 25 subjects from two different sessions with at least 15 days of time elapse. The acquisition is carried out along a hallway with three different smartphones: a OnePlus One (LIS3DH accelerometer by ST Microelectronics), a Samsung Galaxy S4 Active (K330 3-axes accelerometer by ST Microelectronics), and a Sony Xperia S (Bosch Sensortec BMA250 accelerometer). Each walk is about 10 steps long with an average number of 1,300 samples. Each session contains two walks per subject per device. After each acquisition, the device is detached and repositioned to add further variations. In summary, each session contains 6 walks per subject, for a total of 12 walks ( 300 walk signals in total). Each walk is annotated with some demographic information (gender and age range, ethnicity is always Caucasian). This dataset is the only one presently providing cross-sensor signals from different smartphones, and this allows for investigating cross-identification approaches. It is worth considering the different capture accuracy of the embedded accelerometers, and the need to somehow normalize signals before matching.

\section{PREPROCESSING TECHNIQUES}

Some signal preprocessing is often used to improve the data quality before further processing.

Denoising enhances the device signal quality. The noise can be static (constant and devicedependent) and dynamic (from slight fluctuations produced by the gait). The two most exploited algorithms are the Weighted Moving Average (WMA) [45] (used in References [22, 33, 35]), and the Wavelet Denoising (WD) [58] (used in References [85, 86]). WMA requires choosing whether to apply the algorithm symmetrically or only forward, and the length of the sliding window (number of values) entering the computation. For WD the key factors are the choice of the mother wavelet and its amplitude. Figure 6(a) shows an example of denoising by wavelet and by WMA. The wavelet is from References [85, 86].

\footnotetext{
${ }^{4}$ https://sites.google.com/a/di.uniroma1.it/biometric-interaction/home/gait-recognition/datasets/bwr-multidevice.
} 
Time interpolation re-samples the signal to have data points at fixed time distance, by selecting only values at suitable points and approximating missing ones. Linear interpolation is the most used one $[21,22,33,35,68-71,101]$. Each cited work chooses a possibly different re-sampling rate, from 100 to $500 \mathrm{~Hz}$, so that there is no evidence of a common standard for this operation, which actually depends on the characteristics of the original signal at hand. Non-linear time interpolation is used in Reference [49], in a version based on piece-wise cubic spline. The different sampling rate and interpolation strategy impede the comparison of the achieved results, since experiments use different datasets.

Amplitude and/or period normalization are further preprocessing procedures. Amplitude normalization reduces the signals within a common range of values. A popular formula is the standardization one: $v_{n}(i)=\frac{v_{o}(i)-\mu\left(v_{o}\right)}{\sigma\left(v_{o}\right)}$, where $v_{n}(i)$ and $v_{o}(i)$ are the normalized and original signal values, respectively, and $\mu\left(v_{o}\right)$ and $\sigma\left(v_{o}\right)$ are the mean and standard deviation of the original signal.

Period normalization reduces signals to the same length (number of samples) by either upsampling (e.g., by interpolation to increase the sampling rate) or down-sampling (e.g., by decimation to decrease the sampling rate) [55]. Examples are References [1, 31, 32, 59, 70, 71, 86]. Different from interpolation, period normalization does not set the samples time distance, but the compression or extension ratio needed to obtain a signal with fixed time length/number of samples: the acquired values are evenly distributed according to their total number and to the desired final length [1,59]. Figures 6(b) and 6(c) show the different effects of these two procedures, also depending on the chosen time interval parameter. Looking at Figure 6 it is possible to appreciate the effect of denoising and interpolation: the signal is regularized but also loses some apparently characterizing elements. However, the reported results in works using the mentioned techniques seem to testify that such elements are redundant in terms of recognition accuracy. It is not possible to draw further conclusions, since, to the best of our knowledge, a thorough comparison has never been carried out. Alternatively, $[85,86]$ normalize cycle length by Dynamic Time Warping (DTW, usually exploited for matching - see Section 6), while [92] exploits self-DTW [56], considering a pseudo-periodic signal.

Further preprocessing techniques deal with the relations/combinations of the three signal axes and how they are used for matching. The magnitude vector is often used instead of the values from the three single axes [21, 74, 101]. For each instant of time $i$, this vector (g-force vector) provides an acceleration value $v_{i}=\sqrt{x_{i}^{2}+y_{i}^{2}+z_{i}^{2}}$, where $x_{i}, y_{i}$, and $z_{i}$ are the values of the original signal at $i$. The main advantage of the magnitude vector is the invariance with respect to the sensor orientation; this aspect is better discussed in Section 7. In fact, the different orientation of the accelerometer may affect the values of the signals over the single axes (see Section 3). A second pro is the dimensionality reduction from 3D to $1 \mathrm{D}$. However, this kind of preprocessing, being a kind of aggregation, causes losing possible relevant peculiar characteristics over single axes. In particular, it always happens that one axis (the dominant one) captures more relevant information than the others, depending on the orientation. As a consequence, also correlation information is lost. This can be observed in practice and is reported in the literature. Two works $[31,32]$ combine the axes using a different $a d-h o c$ formula, with $v_{i}=\arcsin \left(z_{i} / \sqrt{x_{i}^{2}+y_{i}^{2}+z_{i}^{2}}\right)$. Other proposals use either the single dominant axis [22, 86], or two of them [1, 59]. Finally, $[15,16]$ use the three axes as independent data sources and compute results giving different weights to each of them.

Table 2 summarizes the methods discussed so far and the works using them. It also accounts for the use of magnitude or other combinations of the axes values. Normalization is the most frequent preprocessing step, if the signals are not exploited in their original form. Time is the most used normalization factor (see Section 6.1). Amplitude normalization mostly relies on mean subtraction. A few works use both normalization strategies. Most works carrying out interpolation exploit its 
Table 2. Summary of the Preprocessing Techniques Used for Wearable Sensor-based Gait Recognition

\begin{tabular}{|c|c|c|c|c|c|}
\hline \multirow{2}{*}{ Paper } & \multirow{2}{*}{ Year } & \multicolumn{3}{|c|}{ Preprocessing } & \multirow{2}{*}{$\begin{array}{l}\text { Magnitude (M)/ } \\
\text { Other Combinations }\end{array}$} \\
\hline & & Denoising & Interpolation & Normalization & \\
\hline [22] & 2010 & Weighted Moving Average & Linear & - & - \\
\hline [33] & 2008 & Weighted Moving Average & Linear & Time (on cycles) & - \\
\hline [21] & 2010 & Weighted Moving Average & Linear & Time (on cycles) & M \\
\hline$[35]$ & 2010 & Weighted Moving Average & - & Time (on cycles) & - \\
\hline [85] & 2007 & Wavelet Denoising & - & Time & - \\
\hline [86] & 2007 & Wavelet Denoising & - & Amplitude $([-1,1])$ and Time & - \\
\hline [72] & 2007 & $\begin{array}{l}\text { Direct Form II Transpose filter with a } \\
\text { Gaussian window }\end{array}$ & - & - & - \\
\hline$[28]$ & 2016 & Low Pass Finite Impulse Response filter & Cubic Spline & Time & - \\
\hline$[38]$ & 2017 & Low Pass Butterworth filter (on cycles) & Linear & - & - \\
\hline$[68]$ & 2011 & - & Linear & Amplitude (Mean Subtraction) & - \\
\hline [70] & 2011 & - & Linear & Amplitude (Mean Subtraction) & - \\
\hline [71] & 2012 & - & Linear & Amplitude (Mean Subtraction) & - \\
\hline [69] & 2011 & - & Linear & - & - \\
\hline$[101]$ & 2015 & - & Linear & - & M \\
\hline [49] & 2012 & - & Piece-wise Cubic Spline & Time & - \\
\hline [74] & 2009 & - & - & Time & $\mathrm{M}$ \\
\hline$[32]$ & 2006 & - & - & Amplitude (Mean Subtraction) & $R_{i}=\frac{Z_{i}}{\sqrt{x_{i}^{2}+y_{i}^{2}+z_{i}^{2}}}$ \\
\hline [59] & 2005 & - & - & Amplitude and Time & - \\
\hline [1] & 2005 & - & - & Amplitude and Time & - \\
\hline [25] & 2016 & - & - & - & M \\
\hline
\end{tabular}

linear form. Finally, WMA is the most used method for denoising, if present. The table does not report any performance measure, since different matching algorithms and datasets are used in the experiments, and no results are presented in the papers comparing with vs. without preprocessing.

\section{RECOGNITION METHODS}

A possible classification of state-of-the-art recognition methods includes two main categories. The proposals in the first one generally use signal matching algorithms. Manhattan or Euclidean distances can be used to compute a score, but they can be dramatically affected by time misalignment, especially without any preprocessing. Therefore, they are mostly applied as distance measures for DTW and its variations. Some works preliminarily segment the signals to match into either steps/cycles or fragments/chunks, while a few ones deal with unsegmented signals. Though providing good results, the latter have the limitation to require a sufficient similarity of the lengths of the probe and the gallery walks. In fact, though devised just to match signals of different length by aligning them, also DTW often achieves poor recognition results if the length difference is too large. For this reason, most methods in this first category use some kind of segmentation. Afterward, DTW or similar algorithms are used to match the detected steps/cycles following different strategies. The proposals in the second category generally exploit Machine-learning techniques, such as Support Vector Machine (SVM), k-Nearest Neighbour (k-NN), or Hidden Markov Model (HMM). Also these methods can either use the entire signal or its fragments; in this case, a fragment (also referred to as chunk) is a part of the walking signal of a certain fixed length (either in time or in number of samples). It is possible to notice that the term segment is mostly 
referred to as step/cycle, that has a strict relation with gait physiology, while fragments may lack this characteristic.

In the following, it may seem that some works are described in more detail than others. However, two considerations are worth in this respect, both inspired by the attempt to maintain an easy-to-follow conceptual flow. First, the description of some proposals is divided among different sections, since they present procedures that are used in more papers. For instance, [21] and [86] introduce segmentation algorithms that are also used elsewhere. For this reason, these algorithms are extensively described in Section 6.1, which is devoted to reference segmentation techniques. As a consequence, these papers are more shortly presented afterward, as well as the other ones using the same techniques. On the contrary, when specific processing steps are used only in a single paper, they are all described in the possibly single place where that paper is mentioned. As a second consideration, some works use standard well-known methodologies (e.g., DTW) that are, therefore, only mentioned, while others propose more original approaches that are described in more detail.

\subsection{Step/Cycle Segmentation Procedures}

The possible step/cycle segmentation is a non-negligible procedure. This section describes two state-of-the-art cycle segmentation procedures [21, 86], and a step segmentation procedure [16]. They exemplify different possible approaches to the problem and are clearly explained and therefore repeatable. In other cases, the adopted choices are not completely clear.

6.1.1 Cycle Segmentation Based on Cycle Extremes Identification. The segmentation algorithm proposed by Rong et al. [86] is carried out only on the most significant axis, and the results are projected onto the other two axes. The acceleration data are acquired from a sensor attached on the hip. It is worth pointing out that, depending on the sensor orientation, the most significant axis changes. In general, independently from that, the axis that is perpendicular to the ground can be assumed as the principal one. This specific work deals with $Z$ axis. A preprocessing procedure includes normalization to map acceleration data onto the interval $[-1,1]$, and wavelet denoising (Debauchies order 8 as mother wavelet). The phases of the proposed segmentation algorithm are:

(1) search local minima in the $\mathrm{Z}$ signal;

(2) for each local minimum, search the first next value with a different sign (candidate starting/ending points of cycles);

(3) segmentation: each gait cycle includes all samples within four consecutive points found above.

This proposal is quite fast and gives sufficiently accurate results. However, it is error-prone, especially if the signal falls around the zero value many times. This can be due, e.g., to noise.

6.1.2 Cycle Segmentation Based on Cycle Length Estimation. The algorithm proposed by Derawi et al. [21] is made up of two parts, the cycle length estimation, and the segmentation. Preprocessing entails linear time normalization, to have samples at a constant $\frac{1}{100} s$ distance, and noise reduction through the Weighted Moving Average algorithm. Finally, the magnitude vector $(m v)$ is computed by the standard formula, and used by cycle length estimation and segmentation phases.

\section{Cycle Length Estimation:}

(1) choose a random sequence $s w$ of 70 samples approximately in the middle of the $m v$;

(2) compute an array containing DTW distances between $s w$ and all sequences of 70 elements in the entire $m v$;

(3) look for the local minima in this array of distances; 
(4) compute the average cycle length (call it $\gamma$ ) as the average of the distances between each pair of consecutive local minima in the distance array.

\section{Segmentation:}

(1) choose a local minimum in the signal approximately in the middle of the $m v$ (call it $P_{\text {start }}$ );

(2) look for the closest local signal minimum around the point $P_{\text {start }}+\gamma$ (and symmetrically for $P_{\text {start }}-\gamma$ ), using Neighbour Search; each found point is the start/stop of a cycle;

(3) repeat the search in both directions until to reach the end and start of the signal, respectively.

Cycle length estimation is smart and flexible yet computationally demanding. The segmentation can sometimes not work correctly, because it relies on a fixed number of samples, that could be rather chosen depending on the sampling rate of the accelerometer. Being a cycle composed by two steps, it may happen that a user walk starts from a cycle with left step first, while in a different walk by the same user, the right step comes first (and vice versa). Unless controlled during acquisition, this can create not negligible problems in the recognition phase.

6.1.3 Step Segmentation Based on the Estimated Number of Steps. The BWR procedure (from Biometric Walk Recognizer) [16] is carried out only on the y-axis (the principal axis for this system). As for 6.1.1, the results are then projected onto the other axes. BWR does not exploit preprocessing. However, it needs the (possibly approximate) knowledge of the number of walk steps (call it $k$ ).

\section{Key Variables Estimation:}

(1) compute the average value of the entire signal (call it $m$ ) and the frequency of each value;

(2) select stepEquilibrium as the value with the highest frequency that is lower than $m$; this value is used to avoid an erroneous segmentation caused by noise;

(3) find all the relative maxima and store their locations and values in a list in descending order;

(4) select stepThreshold as the $k$-th highest relative maximum in the list.

\section{Step Segmentation:}

(1) look for the first relative maximum in the signal; it will be the starting point of the first step;

(2) scan the following walk data looking for the first value lower than stepEquilibrium;

(3) look for the next relative maximum greater than stepThreshold; it will be the ending point of the current step and the starting point for the next one;

(4) repeat from (2) till to the end of the entire walk signal.

Note: if a local maximum is found after another without passing through a value lower than stepEquilibrium, this maximum will be discarded, classifying it as noise.

This procedure shows good performances and flexibility, it is not computationally demanding, and it can be applied directly to the raw data, without any preprocessing. However, a priori knowledge of the $k$ value (the number of steps) can seem too limiting. In the context of Reference [16], this is not a serious problem, because the number of steps is needed only for segmenting the gallery templates, that can be assumed to be acquired in a more controlled setting. The values for stepEquilibrium and stepThreshold are stored in the gallery for each walk. For the following probe matching, the key variable estimation is substituted by a kind of data-fitting procedure, which entails matching with a gallery walk after applying the corresponding stored values to segment the 
probe signal. If the subject is the right one, then the segmentation will be better and the comparison will return a higher score. A Kalman filter can also be used to implement a step counter, to estimate $k$ before the segmentation procedure. Finally, it is possible to devise an automatic trigger/ stop procedure driving the signal acquisition, e.g., by radio signals issued by Bluetooth beacons positioned in fixed points. In this case, the length of the walk will be approximately the same in space at least, even if the actual number of steps may vary with the bodily features of the walker [17].

\subsection{Systems Matching Step/cycle-segmented Signals}

This section presents some works in the state-of-the-art that rely on a preliminary segmentation phase. From now on, outliers are defined as those steps/cycles that are too different from the others (according to some threshold), possibly due to noise or punctual temporary variations. Unless differently specified, reported experimental results are obtained in verification operations.

The system in Reference [22] uses a low sampling rate accelerometer, embedded in the Google G1 phone. The gait signal is acquired with a round walk in a plain hallway of $37 \mathrm{~m}$, with a stop of $2 \mathrm{~s}$ within roundtrip, to consider two separate walks. This work precedes Reference [21], cited above. The cycle detection and signal segmentation procedures rely on the use of an average cycle length (ACL) value, which is experimentally estimated here as 40-60 samples (but this would correctly work only for an accelerometer with the same sampling rate). Afterwards, the segmentation proceeds as in Reference [21] (see Section 6.1.2). The system computes the average cycle to use it as a feature vector and applies the DTW algorithm for matching. The procedure only processes the $\mathrm{x}$-axis, because, with the orientation of their device (the long side parallel to the floor) it is the dominant one. This system achieves EER $=20 \%$ using a dataset with 51 subjects, each with two roundtrip sessions.

An evolution of the previous work can be found in Reference [21]. The authors use a better (and dedicated) accelerometer (the Motion Recording 100). Moreover, the preprocessing phase is improved by a noise reduction algorithm and by detection and removal of outlier steps. The step segmentation algorithm used is the one described in Section 6.1.2. For outlier removal, the system computes the DTW distance among all cycle pairs belonging to the same walk signal. Then, the average distance of each cycle from all the others is computed, and finally, the procedure discards all cycles whose average distance is not in the $\mu \pm 2 \sigma$ interval, where $\mu$ is the average of the average distances, and $\sigma$ is their standard deviation. All the remaining cycles are used to build the gait template. In recognition phase, the matchings between cycles in the probe template and cycles in the gallery template are carried out using a cross-comparison strategy that exploits a Cycle Rotation Metric (CRM). In a first step, using Manhattan distance, the most similar probe cycle is found for each gallery cycle, and then DTW is computed for the best probe cycle/gallery cycle pair. This decreases the computational burden. With respect to the previous work, the performance improves to an EER $=5.7 \%$ on a dataset of 60 subjects with 12 gait acquisitions each, collected in two different days.

The proposals in References [85, 86] present and exploit the segmentation algorithm described in Section 6.1.1. In the first one, the recognition relies on a time warping network, based on DTW. The reported results are $\mathrm{RR}=70 \%$ (identification) and $\mathrm{EER}=6.7 \%$ (verification). In the second work, the recognition is performed both in time and in frequency domain. Time domain analysis exploits a DTW-based algorithm achieving EER $=5.6 \%$, while in frequency domain EER $=21.1 \%$.

The work in Reference [33] and its evolution in Reference [35] face the problem related to the different shoes worn by the subjects. The experiments are carried out with four models of shoes with different weights. The same dataset is used for both works and collects 4 walk signals for each shoe model from 30 male subjects along a $20 \mathrm{~m}$ hallway, for a total of 16 walks per subject. Both 
works apply a preliminary interpolation procedure to re-sample the signal at a fixed frequency and use the Weighted Moving Average filter to reduce the level of noise. The part of the signal before the true start of walking motion is discarded by using a fixed threshold on the acceleration value, and symmetrically for the end of the motion. As claimed for other works, this could limit the portability of the system. Signal segmentation only relies on the up-down axis (here it is $\mathrm{x}$ ), using three different fixed thresholds to find the starting and ending points of cycles. In the first proposal, the authors use the average cycle as a gait template and then use the Euclidean distance as a comparison strategy. In the second proposal, instead, they keep all cycles. In this case, the matching strategy is based on cross-comparisons, again based on Euclidean distance, and only the minimum result is taken as a distance score. The second approach seems to show a global improvement in terms of performance, achieving an EER down to $1.6 \%$ in the best scenario. A first aspect highlighted by these works is that in a mixed scenario involving all kinds of shoes, the results decrease drastically to EER $=16.4 \%$ in the best test scenario. As a second one, it is possible to notice that the higher is the weight of the shoes the lower the performance, passing from an EER of $1.6 \%$ to $6.1 \%$.

An original cycle segmentation algorithm is presented in Reference [25] and tested on an inhouse dataset with four different recognition strategies. The in-house dataset collects gait signals from 15 subjects (10 males, 5 females), acquired with a Samsung Galaxy Note 4 positioned in the hip zone and attached to a belt, with the long side parallel to the ground. Each walk is captured along a 40m hallway in three different moments of the day (morning, mid-day, and late afternoon) during two sessions. All subjects are required to wear sweatpants and the same model of shoes. If this, on one hand, improves the homogeneity of the data, then, on the other hand, it is not suitable for a real system where the users are generally free to wear anything. The system performs a cycle detection procedure based on high peaks identification. First, the procedure finds all local maxima. Then removes all those below the maxima average, re-computes a new maxima average, and eliminates again all the maxima below such value. Finally, it discards all maxima that do not have a distance of at least $800 \mathrm{~ms}$ among them. After that, only the 10 cycles with the lowest average distance from the others are kept. The recognition strategies all exploit well-known matching methods. The first one uses the Euclidean distance and achieves an up to 26.67\% EER. The second one uses DTW (up to 28.07\% EER). The third one is Manhattan Rotation. Manhattan distance is used to compare a template with all the possible rotations of another template, and the best result is kept as their distance. This strategy achieves an up to $16.49 \%$ EER. The last comparison is with the CRM, presented in Reference [21] and previously discussed in this section. This procedure achieves an up to $16.38 \%$ EER.

The system presented in Reference [31] uses two different kinds of approaches to recognition. The walks by 21 users are collected with AVR Buttery Accelerometer-Motion Recording 100 along a 70m hallway, and manually divided into two segments (probe and gallery). Both proposed recognizers use the same preprocessing in which the values from each axis are combined as $v_{i}=\arcsin \left(Z_{i} / \sqrt{X_{i}^{2}+Y_{i}^{2}+Z_{i}^{2}}\right)$, producing a mono-dimensional signal. The first matching strategy uses histogram similarity, where histogram bins correspond to quantized values of the original signal. This matcher achieves EER $=5 \%$. The second approach uses cycle group comparison, i.e., comparison of all cycles in a walk. To identify a cycle, it uses the 0 -cross points to segment the signal. This, in general, is not guaranteed to work properly, especially with noisy data. The recognition is performed by matching signals cycle by cycle, and the final score is the number of cycle pairs matching values that meet a fixed threshold. This strategy achieves $9 \%$ of EER.

The authors of Reference [49] present a proposal for gait recognition that exploits the gyroscope too. The data acquisition is performed by an Android smartphone with built-in accelerometer and gyroscope (the model is not specified). The device is positioned in the right pocket with the phone 
facing outwards and oriented vertically. The dataset collects walk signals from 36 users along a hallway of about $25 \mathrm{~m}$ in a roundtrip pattern, at different speeds. After the acquisition, the raw data are re-sampled at $2 \mathrm{~ms}$ intervals (or $500 \mathrm{~Hz}$ ) with piece-wise cubic spline interpolation. Then, the signal is segmented into cycles exploiting the high peaks as starting and ending points. The recognition phase exploits continuous wavelet transform time frequency spectrogram analysis for feature extraction and ciclostationarity analysis for matching. Results are also provided for crossspeed matching. The tests achieve $99.4 \%$ of verification rate at $0.1 \%$ of FAR for pace walk vs. pace walk, $96.8 \%$ for fast walk vs. fast walk, and $61.1 \%$ for pace walk vs. fast walk. These results are very good but it is worth noticing that this system uses the gyroscope too.

The work in Reference [1] describes a system based on the concept of gait_code. The used benchmark is a dataset of 36 users (two sessions per user with three walks each, captured 5 days apart). The procedure stems from the fact that is not interesting to distinguish the right from the left steps, but rather to take into account that the signals that they produce are different. Therefore, it is sufficient to classify the two categories of steps as $a$ steps and $b$ steps. The gait_code is a 4-tuple, composed of the average of $a$ steps and the average of $b$ steps over two out of the three accelerometer axes. This leaves out the averages over the less significant axis, which in this work is identified as $\mathrm{Y}$, therefore producing gait_code $=\langle x a, x b, z a, z b\rangle$. In the recognition phase, the signals are matched taking the best correlation of probe and test codes, considering the possible correspondences between probe and test code pairs of components on the same axis. This strategy produces $6.4 \%$ of EER Variations of this approach are presented in Reference [59]. The walk signals are acquired from 36 users at three different speeds (slow, normal, and fast). A first variation entails signal preprocessing by Fast Fourier Transform (FFT) after dividing the signal into frames 256 samples long and with an overlap of 128 samples. The first 40 FFT coefficients per channel are chained to form a feature vector used for matching. The second variation entails computing the feature vectors by the chaining of 10-bin histograms (normalized by signal length) of accelerations over $x$ and $z$ axes. A final variation entails concatenating third and fourth-order moments for both axes. The achieved EER are, respectively, of 7\% (with the correlation-based method from the previous work), 10\% (FFT coefficients), 19\% (histograms), and 18\% (moments). It is worth underlining that recognition of the right user but with a different level of speed is considered as a wrong answer.

An approach based on Signature Points (SPs) is described in Reference [74]. The idea behind the SPs is to search for informative points in gait acceleration signals and takes inspiration by SIFT for 2D images. Data are collected with five Wii Remote (from the well-known console) located in different body locations. These sensors have lower accuracy than the others. The dataset used for experiments contains 30 subjects with six walks each in two sessions (12 walks total per subject). Acceleration data are combined in the magnitude vector form, using the standard formulation. After that, the walk signals are segmented into cycles (but the used procedure is not explained) and then normalized in length. Finally, the proposed procedure extracts the SPs, corresponding to the positions of the starting and ending points of gait cycles. The positions of such points are used for recognition, with majority voting. The proposed system achieves up to $74.5 \%$ of RR with a single accelerometer (the one located in pelvis zone) and a $96.7 \%$ of RR combining all five accelerometer signals.

Machine-learning techniques are applied in Reference [72] on a preliminarily segmented signal. Acceleration data are collected using the Wii Remote controller in three different walk modalities: straight-line, circuitous and multi-directional, and straight-line with a distorted speed. The dataset includes only seven subjects (up to three acquisitions each for each kind of walk). The cycle segmentation is done taking as starting and ending points the relative maximum values in the signal after the application of the Direct Form II Transpose filter. The recognition is done by 
three different machine-learning techniques, namely, k-NN, Naïve Bayes and Quadratic Discriminant Analysis, and achieves an up to $95 \%$ of accuracy with k-NN, which is generally the best, on straight-line walks, where all methods work obviously better. However, this is obtained with a very small dataset.

Two other proposals exploiting segmentation are presented in Reference [15] and its evolution in Reference [16]. In these works, data are collected using a One Plus One smartphone in a hallway and the recordings contain walks of about 10 steps. In the first work, the dataset includes 26 subjects with three walks each. The dataset is extended to 30 subjects for the second work. No preprocessing algorithm is applied to the original signals, except for a "purification" from the starting and ending portions. These are mostly flat and therefore not significant, due to the manual user operation required by the presented prototype to start and stop signal capturing. The exploited step segmentation procedure is the one described in Section 6.1.3. In addition, similarly to Reference [21], an outlier removal is carried out, but the procedure considers as outliers only those steps that have an average distance from the others higher than $\mu+\sigma$. Since distances are considered, if a step is very similar to the others (average distance lower than $\mu-\sigma$ ), it is rather maintained, differently from other works. The authors use five different matching procedures, all based on the basic formulation of the DTW algorithm. The best performing one, namely, WALK, uses the entire signal without step segmentation, but it is constrained by requiring signals with more or less the same number of steps. ALL STEPS VS. ALL method matches each step of the probe with each step of the gallery template, and takes the average of the best matches as the final value, achieving the second best result. In the first work, the WALK method achieves RR = $95 \%$ and EER $=7.69 \%$, while ALL STEPS VS. ALL method achieves RR $=88 \%$ and EER $=10.46 \%$. In the second work, the proposed segmentation method is compared with the ones described in Sections 6.1.1 and 6.1.2. For the experiments, the walks from both the proprietary dataset (with 4 more users w.r.t. the previous work) and from the two largest datasets OU-ISIR and ZJU-gaitacc (see Section 4) are segmented with the compared methods. The results show a general recognition improvement using the proposed segmentation and data-fitting strategy.

The work in Reference [28] presents IDNet, an authentication framework based on Convolutional Neural Networks (CNN). The CNN is used as a feature extractor, and then One-Class SVM (OSVM) is used for verification. The experiments exploit an in-house dataset with data from 50 subjects collected during six months. Data are acquired using different smartphone models (namely, Asus Zenfone 2, Samsung S3 Neo, Samsung S4, LG G2, LG G4, and Google Nexus 5) positioned in the right front pocket of trousers. Subjects are asked to walk at their normal pace in different walking sessions of about $5 \mathrm{~min}$. Information from accelerometer, gyroscope, and magnetometer are stored in the walking file (magnetometer data are not used in the recognition process). The preprocessing phase exploits cubic spline interpolation to $200 \mathrm{~Hz}$ and denoising by a low pass Finite Impulse Response (FIR) filter with a cutoff frequency of $40 \mathrm{~Hz}$. The walking cycles are extracted from the walk magnitude vector, after low-pass filtering with a cutoff frequency of $3 \mathrm{~Hz}$. Afterward, the minima are found and refined to better fit with the original signal. An orientation invariant transformation is then applied to the cycles identified, using the values taken from the original signal. The transformation maps all data onto a vector space defined by three identified invariant versors. Finally, data are normalized both in time (with spline interpolation to reach 200 samples per cycle) and in amplitude (with the standard Gaussian normalization). A CNN is trained for feature extraction using data from 35 out of the 50 users. The training uses as input the extracted and normalized cycles. The $\mathrm{CNN}$ is made up of two convolutional layers followed by a max pooling layer and two fully connected layers. 10 different networks are trained to find out the best parameters. After feature extraction, an OSVM is trained for each of the remaining 15 users with the derived feature vectors, using part of the cycles from their walks. Each out of the 15 users is 
taken as a target in turn (and the other 14 are impostors), and then the results are averaged. The best OSVM configuration achieves FAR and FRR less than $0.15 \%$ with the appropriate parameters. The authors also investigate how many walking cycles are needed for a reliable authentication, and report that using less than five cycles can be sufficient to recognize the target or reject the probe in the $80 \%$ of the cases. Finally, the use of gyroscope in addition to accelerometer is tested, providing a slight improvement.

Deep convolutional neural networks are exploited in Reference [38]. Experiments are carried out on the ZJU-gaitacc dataset, already mentioned and described in Section 4. It is worth reminding that the dataset contains six walks per user session, with signals coming from five different accelerometers. The processing phase consists of three parts: cycle extraction, filtering, and normalization. The cycle extraction is carried out exploiting the strong changes of values (peaks) on the z-axis occurring when the heel impacts the ground, which causes peaks within the magnitude vector. For noise reduction and enhancement of signal quality, the cycles are filtered through a low pass Butterworth filter and normalized by linear interpolation. The exploited deep network consists of two layers of convolution, followed by a single max pooling and two fully connected layers, finally followed by a softmax layer used to estimate the template class. The classification system has been implemented through the Caffe framework. The tests have used five of the six walk signals per subject as a training set and the remaining one for testing. Only a single session in the dataset has been used. To improve the performance of the deep learning network and to prevent overfitting, synthetic training samples are added. This data augmentation consists of the application of one or more deformations to the labeled data without changing the meaning of the labels. The re-identification accuracy of the proposed scheme is measured as the mean number of cycles recognized correctly for each identity as the first, second and third similarity results returned from the network. The authors report $0.94 \%$ of accuracy without augmentation and a little improvement up to $0.95 \%$ of accuracy with data augmentation. It is worth pointing out that the paper probably reports an error, because the correct values seem to be either $94 \%$ and $95 \%$, respectively, or 0.94 and 0.95 , respectively.

\subsection{Systems Matching Timed Chunks of Signal}

The procedures presented in this section do not rely on a preliminary segmentation phase. Rather, fragments of the signals to match are determined by some different strategies.

Reference [52] deals with accelerometer signals captured by a smartphone. The raw time series are first transformed into "examples," because the exploited classification algorithms [97] do not process time series. Experimental data belongs to 36 volunteers carrying an Android smartphone in their front pants pocket while performing a specific set of activities, namely, walking, jogging, climbing upstairs, and climbing downstairs for specific periods of time. Data collection is stopped when the subject switches activities. The data is divided into 10s segments (referred to as example duration (ED)). Afterward, 43 features are extracted from the accelerometer values of each segment (600 total acceleration values per segment, 200 per axis, given by sampling 20 times per second for the 10s interval). Features are mostly computed on a per axis basis and are variations of six basic ones: Average, Standard Deviation, Average Absolute Difference, Average Resultant Acceleration, Time Between Peaks, Binned Distribution. Details on the measures can be found in the paper. Data is used to generate six distinct datasets. Four of them contain examples separated by activity, to assess their effect on recognition. An "aggregate" dataset merges examples from all four activities with the activity label removed (the most realistic scenario). The last "aggregate (oracle)," is identical to the "aggregate" but contains the activity labels, providing information on the utility of knowing the class label (preliminary activity detection). Decision Trees and Neural Networks are used to train single user models and for recognition. Authentication results seem 
missing. Identification results are also reported on a per-user basis and seem to be evaluated in homogeneous conditions, except for the aggregate dataset. The walking and jogging data show the best performance. Ascending and descending stairs provide significantly lower accuracies, but there are much fewer data available for building the models.

The works in References [68, 69] provide two solutions for gait recognition, both tested on the same dataset of 48 subjects with four walks each, collected throughout two sessions with a Google G1 phone. In the first work, the data are re-sampled at $200 \mathrm{~Hz}$ and then divided into fragments (walk sections) of $3 \mathrm{~s}$ without overlap (for sake of homogeneity, 28 fragments are stored per person). For each subject, an HMM is trained using 20 such fragments as positive examples, while the remaining fragments are used for testing. For each HMM, the set of negative samples includes 840 fragments taken from 30 subjects (that will not be included in testing). A test is carried out for each HMM/user, to imitate the situation of a single enrolled user, i.e., the owner of the phone. Testing is carried out with the remaining 8 fragments as genuine samples, and 476 samples from the 17 subjects not involved in training as impostor samples. The experiments exploit the HVITE tool and each subject is used one time as genuine and forty-seven times as negative training/impostor. This strategy reports an EER of about $10 \%$. In the second work, the signals are interpolated at $100 \mathrm{~Hz}$ and divided into fragments (segments) of 7 seconds with a 50\% overlap. Since SVM is used for classification, the acceleration data from each fragment is transformed into a feature vector of fixed size. Features are mostly statistical ones, e.g., mean, maximum, minimum, binned distribution, and so on, with the addition of both the Mel and the Bark frequency cepstral coefficients. Radial Basis Function (RBF) kernel is chosen for the SVM. Walks from the first day are used for training and those from the second day for testing. The presented strategy initially achieves a very high FNMR (above 61\%) while the FMR is reasonable (above 1\% but below 2\%) for all tested configurations (e.g., subsets of features or acceleration axis). To reduce FNMR, the system exploits a quorum voting (at least a certain number of positive responses must be obtained for the probe to be accepted) using all test fragments of a user, treating each of them as an independent test. The same authors had experimentally demonstrated that this strategy works better than majority voting when the difference between FMR and FNMR is very high. In fact, the system achieves a down to $5.9 \%$ FMR and a down to $6.3 \%$ FNMR.

In Reference [71] a k-NN approach is used. The walk signals are collected from 36 subjects during two sessions made up by 12 walks at normal pace, 16 walks at fast pace and further 12 walks at normal pace on a flat hallway. Walks are separated by stop periods (when the subject is not walking) by an automatic procedure and then manually corrected when necessary. The extracted walking signals are interpolated at $127 \mathrm{~Hz}$. Afterward, they are divided into fragments. Each round of tests uses a different fragment size (3s, $5 \mathrm{~s}$, and $7.5 \mathrm{~s}$, respectively) always with a $50 \%$ overlap. Similar to Reference [68], the feature vectors contain some statistical parameters and both $\mathrm{Mel}$ and Berk coefficients extracted from the three axes and the magnitude vector. The matching exploits the k-NN algorithm included in the WEKA library. As in Reference [68], a quorum voting approach is introduced to reduce FNMR. The best reported performance among the tested configurations is EER $=8.24 \%$

The work in Reference [54] proposes a system where the mobile acquisition device can be kept anywhere. This approach presents some peculiar aspects and, therefore, it will be described in more detail.

Three datasets are collected (using Samsung Galaxy S3, S4, Google Nexus 5, and Intel Xolo) for different aims. The first one contains data from 47 subjects (19 females and 28 males), naturally performing different activities with the same frequency while keeping the phone in different body placements. These acquisitions sum up to $18 \mathrm{~h}$ of data. This dataset is used to train a walking detector, determining whether the input frame is a walking one. The same dataset is used also 
to train a Gaussian Mixture Model-Universal Background Model (GMM-UBM), representing a subject-independent distribution of gait patterns under various conditions. Two alternatives are tested for a second step of training, either supervised or unsupervised, to recognize single subjects from more specific features. The supervised alternative is evaluated using the second collected dataset, with data from 12 subjects ( 5 females and 7 males), with the phone in at least 2 placements (e.g., pant front pocket, jacket pocket, and bags), in multiple sessions and at different speeds. Data is annotated accordingly. These acquisitions sum up to about 1 hour of walking data per subject. For each user, $1 / 3$ of the data is used to learn the individual gait model and the rest for testing. The third dataset is used for evaluating the unsupervised version of the second step of training. Unlabeled accelerometer data are collected 24/7 from eight subjects during two or three weeks. On average, the mentioned walking detector returns a total of $5 \mathrm{~h}$ of data per subject.

All datasets undergo the same preprocessing phase. Accelerometer data are fragmented into frames of 512 samples each with an overlap of 50\%. All stationary frames are discarded using an energy threshold. After this first selection, a low-pass mean filter is applied to each sample in the frame to estimate the direction of gravity, represented as the triplet of average values of, respectively, $\mathrm{x}, \mathrm{y}$, and $\mathrm{z}$ axes over all samples in the frame. If this triplet changes drastically with respect to the immediately preceding frame, then the current frame is discarded, since it is possible that an abrupt change in device orientation happened. The low-pass filtered remaining points are considered as a vector and remapped into two rotation invariant vectors, one for its vertical component and one for the horizontal one. The frame is represented as the set of component pairs of its samples.

The proposed system extracts a combination of time domain, frequency domain, and autocorrelation features from each frame. A small set of these features is used for walking detection. This is carried out exploiting a decision tree classifier achieving a precision of about $98 \%$ and a recall of about $95 \%$.

After walking detection, the relevant features for gait analysis are extracted from walking frames only. A set of features includes the compressed sub-band cepstral coefficients (CSCC), which is inspired by MFCC (Mel-frequency cepstral coefficients) feature set for audio analysis (used in speech recognition and speaker identification systems). Further features are related to autocorrelation.

The system achieves an EER of about $14 \%$ when $20 \%$ of labeled data is used for user gait training (using the second dataset). With unlabeled data (third dataset), the results get worse by about $5 \%$. On one hand, it seems strange that the difference is not so high, on the other hand, this is probably due to the larger amount of data being exploited to train the system when using unlabeled data.

\subsection{Systems Matching Unsegmented Signals}

One of the main advantages in the use of an unsegmented signal is that, in this case, the coarticulation between the single steps/cycles is maintained, whose effect might be considered similar to the phenomenon observed in speech, where the pronunciation of a vowel or consonant can be influenced by the preceding one [41]. Similarly, we may assume a different step/cycle starting or ending shape according to the continuation of the movement flow characterizing the previous step/cycle. This might especially hold with short walks (see Section 8.2).

The proposal in Reference [101], already mentioned in Section 4, presents an evolution of Reference [74]. The collected data are converted into magnitude vectors using the standard formula. Differently from the previous work, the steps segmentation procedure is substituted by an improved version of the signature points extraction. The signature points are taken as the extrema of the convolution of the gait signal with a Difference of Gaussian pyramid. An extremum is defined as a point that is greater or smaller than all its eight neighbors. These points are then stored as vectors for a sparse representation. Then they are clustered, linearly combined and saved into a 
dictionary, to obtain a single element for each cluster. For recognition, the system considers the matching as a conditional probability problem and uses a sparse-code classifier. The results are very interesting and reach an up to $95.8 \%$ RR (identification), and a down to $2.2 \%$ EER (verification), even if it is worth considering that the approaches in both works use five accelerometers worn in different body locations.

As mentioned in Section 6.2, one out of the five methods presented in Reference [16] (namely, WALK) compares the entire signals using DTW. When executed on data from ZJU-gaitacc captured from the hip accelerometer (the most likely position for a smartphone), it achieves better results than Reference [74].

\subsection{Summary Tables of State-of-the-art Proposals}

Table 3 summarizes the state-of-the-art approaches to gait recognition by wearable sensors. It schematizes the works in the same order as they have been presented in this section, maintaining the subsection subdivision. The only work exclusively exploiting unsegmented walk signals is the last one in the table. For sake of layout organization, details about the datasets used by each work are reported separately in Table 4, maintaining the same ordering of papers for an easiest cross-reference.

Table 3 raises some interesting considerations. State-of-the-art proposals based on fragmentation, at the best of our knowledge, or according to the reviewed literature at least, always use a Machine-learning-based recognition strategy. On the contrary, those that rely on step/cycle segmentation generally, but not always, exploit signal matching strategies based on distances or similarities. Moreover, with the exception of References [15, 16], all works use some preprocessing to improve the quality of the signal and/or to process data of an equal length and/or with a same frequency. As already mentioned in Section 4, and as it is even more evident from Table 4, the majority of the proposals use in-house datasets as test-bed, which (often significantly) differ for number of subjects, acquisition device(s), device(s) position(s) and possibly number, and length of the acquired signals per user. Moreover, some datasets are collected during a single session, and sometimes with data coming from a single walking sequence divided into two or more parts. This is the case, e.g., with the huge OU-ISIR dataset [66]. This factor drastically limits the intra-class differences and makes the recognition task easier than the matching of data really collected in more than one session. For these reasons, though performance measures are reported in the text for readers' interest, their full and significant comparison is not easy and often even unfeasible. Regarding device positioning, the most popular locations are the central parts of the body (namely, the ones described as $\mathrm{Be}=$ belt, $\mathrm{W}=$ waist, $\mathrm{H}=$ hip, and $\mathrm{TP}=$ trousers pocket in Table 4). This is probably due to the observation of the intrinsic physical characteristics of the human body and of gait kinematics, that make this zone a privileged center of gravity and therefore a good candidate for acquisition. Moreover, they are the more realistic positions for a smartphone, especially for the TP. Another interesting factor in Table 3 is that most works test the proposed methodology in verification operation, assuming the owner of the device as the implicitly claimed identity. Even though identification operation is sometimes reported, it is always closed set except for the experiments in Reference [16]. This trend is quite common to most biometrics, since open set identification usually dramatically affects performance in a negative way (see Section 2). However, this kind of operation is the most realistic, too, therefore recent works are using it $[39,96]$.

The results of the proposed recognition strategies, even if not comparable, are generally quite interesting, especially considering that gait is classified as a soft biometrics. This makes it a good candidate for complementing other hard biometrics. However, it is to say that extensive datasets and evaluation are still missing for completely uncontrolled conditions and for massive variations regarding ground slope, shoe type, and other sources of variation that may affect gait signal. It 
Table 3. Surveyed Methods Divided by Recognition Strategies

\begin{tabular}{|c|c|c|c|c|c|c|c|c|}
\hline \multirow[b]{2}{*}{ Paper } & \multirow[b]{2}{*}{ Year } & \multicolumn{4}{|c|}{ System } & \multirow[b]{2}{*}{ Dataset } & \multicolumn{2}{|r|}{ Results } \\
\hline & & $\begin{array}{l}\text { Machine } \\
\text { Learning }\end{array}$ & Preprocessing & $\begin{array}{c}\text { Methodology/ } \\
\text { Feature Extraction }\end{array}$ & Matching & & $\begin{array}{l}\text { Biometric } \\
\text { Operation }\end{array}$ & Performance \\
\hline$[22]$ & 2010 & NO & $\mathrm{D}, \mathrm{I}$ & $\begin{array}{c}\text { Uses only x-axis, } \\
\text { CS, } \\
\text { ACE }\end{array}$ & DTW & In-house & V & $20.1 \%$ EER \\
\hline$[21]$ & 2010 & NO & $\mathrm{D}, \mathrm{I}, \mathrm{M}$ & $\begin{array}{l}\text { CS } \\
\text { OR }\end{array}$ & $\begin{array}{l}\text { AaA using } \\
\text { CRM with } \\
\text { DTW }\end{array}$ & In-house & V & $5 \% \mathrm{EER}$ \\
\hline [85] & 2007 & NO & $\mathrm{D}, \mathrm{N}$ & $\begin{array}{c}\text { (TD) CS, } \\
\text { (FD) Discrete FT }\end{array}$ & $\begin{array}{l}\text { (TD) DTW, } \\
\text { (FD) FT } \\
\text { coefficient } \\
\text { similarity }\end{array}$ & In-house & V & $\begin{array}{l}\text { (TD) } 5.6 \% \text { EER, } \\
\text { (FD) } 21.1 \% \mathrm{EER}\end{array}$ \\
\hline [86] & 2007 & NO & $\mathrm{D}, \mathrm{N}$ & $\mathrm{CS}$ & DTW with ED & In-house & V CSI & $\begin{array}{c}6.7 \% \mathrm{EER}, \\
\text { about } 70 \% \mathrm{RR}\end{array}$ \\
\hline [33] & 2008 & NO & $\mathrm{D}, \mathrm{I}, \mathrm{N}$ & $\begin{array}{c}\mathrm{CS}, \\
\text { ACE using median }\end{array}$ & $\begin{array}{c}\mathrm{ED}, \\
\text { weighted } \mathrm{ED}\end{array}$ & In-house & V & $\begin{array}{c}7.2 \%-5 \% \text { EER, } \\
5.6 \% \text { EER, } \\
15-12.8 \% \text { EER, } \\
8.3-7.8 \% \text { EER }\end{array}$ \\
\hline [35] & 2010 & NO & $\mathrm{D}, \mathrm{I}, \mathrm{N}$ & $\mathrm{CS}$ & $\begin{array}{l}\text { CrC ED } \\
\text { taking MIN }\end{array}$ & In-house & V & $\begin{array}{l}\text { 1.6\% EER, } \\
2.8 \text { EER, } \\
5 \% \text { EER, } \\
3.3 \% \text { EER }\end{array}$ \\
\hline$[25]$ & 2016 & NO & M & $\begin{array}{l}\text { CS, } \\
\text { keeps the best } 10 \\
\text { cycles }\end{array}$ & $\begin{array}{l}\text { ED, } \\
\text { DTW, } \\
\text { MR, } \\
\text { CRM }\end{array}$ & In-house & V & $\begin{array}{c}\text { ED } 26.67 \% \text { EER, } \\
\text { DTW } 28.07 \% \text { EER, } \\
\text { CRM 16.38\% EER, } \\
\text { MR } 16.49 \% \text { EER }\end{array}$ \\
\hline [31] & 2006 & NO & $\mathrm{N}, \mathrm{O}$ & $\begin{array}{c}\mathrm{CS} \\
\mathrm{H}\end{array}$ & $\begin{array}{l}\mathrm{CC}, \\
\mathrm{HS}\end{array}$ & In-house & V & $\begin{array}{l}\text { HS: } 5 \% \text { EER, } \\
\text { CC: } 9 \% \text { EER }\end{array}$ \\
\hline [49] & 2012 & Both & $\mathrm{D}, \mathrm{I}$ & $\begin{array}{c}\mathrm{CS}, \\
\text { OR, } \\
\mathrm{CWT}(\mathrm{MHW})\end{array}$ & $\begin{array}{l}\text { TFSA using } \\
\text { CA with } \\
3 \text { different } \\
\text { procedures } \\
\text { SVM }\end{array}$ & In-house & V & $\begin{array}{l}99.4 \% \text { VR } \mathrm{n} \text { vs. } \mathrm{n}, \\
96.8 \% \text { VR f vs. } \mathrm{f} \\
61.1 \% \text { VR n vs. } \mathrm{f} \\
\text { at } 0.1 \% \text { FAR }\end{array}$ \\
\hline [1] & 2005 & $\mathrm{NO}$ & $\mathrm{N}$ & $\begin{array}{c}\text { SS, } \\
\text { Uses only } \mathrm{x} \text { and } \\
\mathrm{z} \text { axes }(\mathrm{GC})\end{array}$ & $\mathrm{CrC}$ & In-house & V & $6,4 \%$ EER \\
\hline [59] & 2005 & NO & $\mathrm{N}$ & $\begin{array}{c}\text { SS, } \\
\text { Uses only } \mathrm{x} \text { and } \mathrm{z} \\
\text { axes (GC), FFT } \\
\text { feature, } \mathrm{H}\end{array}$ & $\begin{array}{c}\text { (TD) } \mathrm{CrC} \\
\text { taking MAX, } \\
\text { HS, } \\
\text { HOM, } \\
\text { (FD) FFT } \\
\text { feature } \\
\text { comparison }\end{array}$ & In-house & V & $\begin{array}{l}7 \% \text { EER } \\
\text { NOTE: Right user yet } \\
\text { recognized with the } \\
\text { wrong speed is } \\
\text { considered a wrong } \\
\text { response }\end{array}$ \\
\hline [74] & 2009 & $\mathrm{NO}$ & $\mathrm{N}, \mathrm{M}$ & $\begin{array}{l}\text { CS, } \\
\text { SPs }\end{array}$ & $\begin{array}{l}\text { MV of SPs } \\
\text { label } \\
\text { prediction }\end{array}$ & In-house & CSI & $\begin{array}{c}\text { Wrist } 66.8 \% \mathrm{RR} \text {, } \\
\text { Upper arm } 74.5 \% \mathrm{RR} \text {, } \\
\text { Waist } 70.1 \% \mathrm{RR} \text {, } \\
\text { Thigh } 67.5 \% \mathrm{RR}, \\
\text { Ankle } 72.9 \% \mathrm{RR}, \\
\text { All five } 96.7 \% \mathrm{EER}\end{array}$ \\
\hline [72] & 2009 & $\begin{array}{l}\text { k-NN NB } \\
\text { QDA }\end{array}$ & $\mathrm{D}$ & $\begin{array}{c}\mathrm{CS}, \\
\mathrm{k}-\mathrm{NN}, \mathrm{NB}, \\
\text { and QDA classifier } \\
\text { training }\end{array}$ & $\begin{array}{l}\text { k-NN NB } \\
\text { QDA }\end{array}$ & In-house & V & $\begin{array}{l}\text { up to } 95 \% \text { of accuracy } \\
\text { with k-NN }\end{array}$ \\
\hline [15] & 2015 & NO & - & $\begin{array}{l}\text { SS, } \\
\text { OR }\end{array}$ & $\begin{array}{l}5 \text { methods } \\
\text { based on } \\
\text { DTW }\end{array}$ & In-house & V CSI & $\begin{array}{c}95 \% \text { RR, } 7.69 \% \text { EER } \\
\text { with entire walk } \\
\text { DTW, up to } 88 \% \text { RR } \\
\text { and down to } 10.46 \% \\
\text { EER with SS methods }\end{array}$ \\
\hline [16] & 2016 & NO & - & $\begin{array}{l}\text { SS, } \\
\text { OR }\end{array}$ & $\begin{array}{l}5 \text { methods } \\
\text { based on } \\
\text { DTW }\end{array}$ & $\begin{array}{l}\text { In-house, } \\
\text { ZJU- } \\
\text { gaitacc, } \\
\text { OU-ISIR }\end{array}$ & CSI V OSI & $\begin{array}{c}\text { Comparisons } \\
\text { between SS and } \\
\text { different datasets }\end{array}$ \\
\hline
\end{tabular}


Table 3. Continued

\begin{tabular}{|c|c|c|c|c|c|c|c|c|}
\hline \multirow[b]{2}{*}{ Paper } & \multirow[b]{2}{*}{ Year } & \multicolumn{4}{|c|}{ System } & \multirow[b]{2}{*}{ Dataset } & \multicolumn{2}{|r|}{ Results } \\
\hline & & $\begin{array}{l}\text { Machine } \\
\text { Learning }\end{array}$ & Preprocessing & $\begin{array}{l}\text { Methodology/ } \\
\text { Feature Extraction }\end{array}$ & Matching & & $\begin{array}{l}\text { Biometric } \\
\text { Operation }\end{array}$ & Performance \\
\hline [28] & 2016 & $\mathrm{CNN}$ & $\mathrm{I}, \mathrm{N}, \mathrm{O}$ & $\begin{array}{c}\text { CS, } \\
\text { OIT, } \\
\text { CNN training }\end{array}$ & $\begin{array}{l}\text { CNN-based } \\
\text { Classification }\end{array}$ & In-house & $\mathrm{V}$ & $\begin{array}{c}\text { FAR and a FRR less } \\
\text { than } 0.15 \% \text { with } \\
\text { appropriate } \\
\text { thresholds }\end{array}$ \\
\hline [38] & 2017 & Deep CNN & $\mathrm{D}, \mathrm{N}$ & $\begin{array}{c}\text { CS, } \\
\text { CNN training, } \\
\text { DA }\end{array}$ & $\begin{array}{l}\text { CNN-based } \\
\text { Classification }\end{array}$ & $\begin{array}{l}\text { ZJU- } \\
\text { gaitacc }\end{array}$ & V & $\begin{array}{c}0.94 \%(?) \text { accuracy } \\
\text { (w/o DA) } 0.95 \%(?) \\
\text { accuracy (w DA) }\end{array}$ \\
\hline$[52]$ & 2010 & $\begin{array}{l}\text { Decision } \\
\text { Tree, } \\
\text { Neural } \\
\text { Network }\end{array}$ & $\mathrm{F}$ & $\begin{array}{l}\text { Feature Extraction } \\
\text { Training of } \\
\text { Decision Tree } \\
\text { Training of Neural } \\
\text { Network }\end{array}$ & $\begin{array}{c}\text { Decision Tree } \\
\text { Classification } \\
\text { Neural } \\
\text { Network } \\
\text { Classification }\end{array}$ & In-house & V I & $\begin{array}{l}\text { Results provided only } \\
\text { for single user }\end{array}$ \\
\hline$[69]$ & 2011 & HMM & I & $\begin{array}{c}\text { F, } \\
\text { HMM Training } \\
\text { (with Baum-Welch } \\
\text { algorithm). }\end{array}$ & HMM & In-house & $\mathrm{V}$ & $\begin{array}{c}\text { 10,42\% FNMR, } \\
\text { 10,29\% FMR, } \\
\text { about } 10 \% \text { EER }\end{array}$ \\
\hline$[68]$ & 2011 & SVM & I & $\begin{array}{c}\mathrm{F}, \\
\text { MFCC and BFCC } \\
\text { extraction, SVM } \\
\text { training }\end{array}$ & SVM & In-house & $\mathrm{V}$ & $\begin{array}{c}5,9 \% \text { FMR with } 6,3 \% \\
\text { FNMR }\end{array}$ \\
\hline [71] & 2012 & $\mathrm{k}-\mathrm{NN}$ & $\mathrm{I}, \mathrm{N}$ & $\begin{array}{l}\text { F (3 dimensions), } \\
\text { Feature extraction. }\end{array}$ & k-NN with ED & In-house & $\mathrm{V}$ & $8,24 \%$ HTER \\
\hline$[54]$ & 2014 & GMM & $\mathrm{D}, \mathrm{O}$ & $\begin{array}{c}\mathrm{F}, \\
\text { Walking frame } \\
\text { detection, } \\
\text { Feature Extraction } \\
\text { (TD and FD), } \\
\text { GMM-UBM } \\
\text { training, } \\
\text { GMM training } \\
\text { (specific user) }\end{array}$ & GMM & In-house & $\mathrm{V}$ & $\begin{array}{l}\text { Walk stance detection } \\
\text { accuracy of } 98 \% \text {, } \\
14 \%-20 \% \text { EER labeled, } \\
\text { about } 25 \% \text { EER } \\
\text { unlabeled }\end{array}$ \\
\hline$[101]$ & 2015 & $\begin{array}{l}\text { SPs with } \\
\text { Clustering }\end{array}$ & $\mathrm{I}, \mathrm{M}$ & $\begin{array}{l}\text { SPs extraction, } \\
\text { Sparse } \\
\text { Representation of } \\
\text { SPs, } \\
\text { Clustering }\end{array}$ & $\begin{array}{l}\text { Sparse Code } \\
\text { Classifier }\end{array}$ & $\begin{array}{c}\text { ZJU- } \\
\text { gaitacc }\end{array}$ & CSI, V & $\begin{array}{c}\text { up to } 73,4 \% \text { RR }(\mathrm{H}) \\
\text { and down to } 8,6 \% \\
\text { EER (T), } \\
95.8 \% \text { RR, 2.2\% EER } \\
\text { with all five sensors }\end{array}$ \\
\hline \multicolumn{9}{|c|}{ Legend of acronyms: } \\
\hline \multicolumn{3}{|c|}{$\begin{array}{l}\text { AaA }=\text { All against All } \\
\text { ACE }=\text { Average Cycle Extraction } \\
\text { BFCC=Bark freq. cepstral coeffs } \\
\text { CA }=\text { Cyclostationarity Analysis } \\
\mathrm{CC}=\text { Cycle Comparisons } \\
\text { CrC=Cross Correlation } \\
\text { CRM=Cyclic Rotation Metric } \\
\text { CS }=\text { Cycle Segmentation } \\
\text { CSI }=\text { Closed Set Identification }\end{array}$} & \multicolumn{2}{|c|}{$\mid \begin{array}{l}\text { CWT=Continuous Wavelet Transform } \\
\text { D=Denoising } \\
\text { DTW=Dynamic Time Warping } \\
\text { ED=Euclidean Distance } \\
\text { F=Division into Fragments } \\
\text { FD=Frequency Domain } \\
\text { FT=Fourier Transform } \\
\text { GMM=Gaussian Mixture Model } \\
\text { H=Histogram }\end{array}$} & \multicolumn{2}{|c|}{$\begin{array}{l}\mathrm{I}=\text { Interpolation } \\
\mathrm{k}-\mathrm{NN}=\mathrm{k}-\mathrm{Nearest} \text { neighbors } \\
\mathrm{M}=\mathrm{Magnitude} \\
\text { MFCC=Mel freq. cepstral coeffs } \\
\mathrm{N}=\text { Normalization } \\
\mathrm{NB}=\text { Naive Bayes } \\
\mathrm{O}=\text { Other kind of combination } \\
\text { OR=Outlier Removal } \\
\text { OSI=Open Set Identification }\end{array}$} & \multicolumn{2}{|c|}{$\begin{array}{l}\text { QDA=Quadratic Discriminant Analysis } \\
\text { SA=Spectrogram Analysis } \\
\text { SPs=Signature Points } \\
\text { SS=Step Segmetation } \\
\text { TD=Time Domain } \\
\text { TF=Time Frequency } \\
\text { UBM=Universal Background Model } \\
\text { V=Verification }\end{array}$} \\
\hline
\end{tabular}

is worth pointing out again that most works carry out limited comparisons, if any: they either compare proposals from the same research group, to highlight progress or, quite seldom, they compare similar approaches. On the contrary, the work introducing the large OU-ISIR database [66], compares four benchmark gait-based recognition methods (Rong et al. [85], Gafurov et al. [35], Derawi et al. [21], and Trung et al. [92]). The best results only achieve $14.3 \%$ EER, but it is to consider that the gait signals are very short (see Section 4) so that there is no beneficial effect due to the natural gait stabilization effect reported for longer walks (see References [25, 60] in Section 8.1).

\section{GAIT RECOGNITION IN LESS CONTROLLED CONDITIONS}

This section summarizes some outcomes related to a less controlled acquisition of the gait signal. 
Table 4. The Main Characteristics of the Datasets Exploited in the State-of-the-art Works

\begin{tabular}{|c|c|c|c|c|c|c|c|c|}
\hline \multicolumn{2}{|c|}{ Data } & \multicolumn{7}{|c|}{ Dataset } \\
\hline Paper & Year & Device(s) & $\begin{array}{c}\text { Device(s) } \\
\text { Position(s) }\end{array}$ & Subjects & $\begin{array}{l}\text { Walk } \\
\text { Length }\end{array}$ & $\begin{array}{l}\text { \#Walks per } \\
\text { subject in } \\
\text { each session }\end{array}$ & \#Sessions & Notes \\
\hline$[22]$ & 2010 & $\begin{array}{c}\text { Google G1 } \\
(\mathrm{AK} 8976 \mathrm{~A}) 50 \mathrm{~Hz}\end{array}$ & R.H. & $\begin{array}{c}51 \\
(41 \mathrm{M}-10 \mathrm{~F}) \\
\end{array}$ & $37 m+37 m$ & $\begin{array}{l}2 \text { (from the } \\
\text { same walk) }\end{array}$ & 2 & - \\
\hline [21] & 2010 & $\begin{array}{c}\text { Motion Recording } \\
100 \mathrm{~Hz} \\
\end{array}$ & Be (L.H.) & 60 & $20 m+20 m$ & 6 & 2 & - \\
\hline [85] & 2007 & \multirow[t]{2}{*}{ MMA7260 } & \multirow[t]{2}{*}{ B.W. } & $\begin{array}{c}21 \\
(11 \mathrm{M}-10 \mathrm{~F}) \\
\text { age }[19,40]\end{array}$ & \multirow[t]{2}{*}{$30 \mathrm{~m}$} & \multirow[t]{2}{*}{5} & \multirow[t]{2}{*}{1} & \multirow[t]{2}{*}{$\begin{array}{c}1 \text { per user for training } \\
\text { and } 4 \text { for test (randomly } \\
\text { chosen) }\end{array}$} \\
\hline [86] & 2007 & & & $\begin{array}{c}35 \\
(19 \mathrm{M}-16 \mathrm{~F}) \\
\text { age }[20,45]\end{array}$ & & & & \\
\hline [33] & 2008 & \multirow{2}{*}{$\begin{array}{l}\text { Motion Recording } \\
100 \mathrm{~Hz}\end{array}$} & \multirow{2}{*}{ R.A. } & \multirow{2}{*}{$30(30 \mathrm{M})$} & \multirow{2}{*}{$20 \mathrm{~m}$} & \multirow{2}{*}{$\begin{array}{l}4 \text { (per shoes } \\
\text { model) }\end{array}$} & \multirow{2}{*}{1} & \multirow{2}{*}{4 different shoes model } \\
\hline$[35]$ & 2010 & & & & & & & \\
\hline$[25]$ & 2016 & $\begin{array}{l}\text { Samsung Galaxy } \\
\text { Note } 4100 \mathrm{~Hz}\end{array}$ & $\mathrm{H}$ & $\begin{array}{c}15 \\
(10 \mathrm{M}-5 \mathrm{~F}) \\
\text { age }[21,38]\end{array}$ & $40 m+40 m$ & $\begin{array}{l}6 \text { (different } \\
\text { day time) }\end{array}$ & 2 & $\begin{array}{c}\text { Same shoes model for } \\
\text { all subjects, all subjects } \\
\text { wear sweatpants }\end{array}$ \\
\hline [31] & 2006 & $\begin{array}{l}\text { AVR Butterfly } \\
\text { (ADXL202) } 16 \mathrm{~Hz}\end{array}$ & R.A. & $\begin{array}{c}21 \\
(12 \mathrm{M}-9 \mathrm{~F}) \\
\text { age }[20,40]\end{array}$ & $\begin{array}{c}35 \mathrm{~m}+35 \mathrm{~m} \\
\text { manually } \\
\text { divided }\end{array}$ & $\begin{array}{l}2 \text { (from the } \\
\text { same walk) }\end{array}$ & 1 & - \\
\hline [49] & 2012 & $\begin{array}{l}\text { Not specified } \\
\text { Android device }\end{array}$ & R.TP & $\begin{array}{c}36 \\
(28 \mathrm{M}-8 \mathrm{~F})\end{array}$ & $25 m+25 m$ & 3 pace +3 fast & 1 & 2 levels of speed \\
\hline [1] & 2005 & \multirow{2}{*}{ ADXL202JQ 256Hz } & \multirow{2}{*}{ B.C.W. } & \multirow{2}{*}{$\begin{array}{c}36 \\
(19 \mathrm{M}-17 \mathrm{~F})\end{array}$} & \multirow{2}{*}{$20 \mathrm{~m}$} & \multirow{2}{*}{3} & \multirow{2}{*}{$\begin{array}{l}2 \text { (5 days } \\
\text { delay) }\end{array}$} & \multirow{2}{*}{-} \\
\hline$[59]$ & 2005 & & & & & & & \\
\hline$[74]$ & 2009 & Wii Remote $100 \mathrm{~Hz}$ & $\begin{array}{l}\text { L.Ar., } \\
\text { L.Wr., R.H., } \\
\text { L.T. R.A. } \\
\end{array}$ & 30 & $20 \mathrm{~m}$ & 6 & $\begin{array}{l}2 \text { (from } 1 \text { to } \\
2 \text { months } \\
\text { delay) }\end{array}$ & - \\
\hline [72] & 2009 & $\begin{array}{c}\text { Wii Remote + } \\
\text { Motion Plus (gyro) } \\
50 \mathrm{~Hz}\end{array}$ & $\mathrm{TP}$ & 7 & $\begin{array}{l}\text { not } \\
\text { reported }\end{array}$ & $\begin{array}{l}\text { up to } 3 \text { times } \\
\text { per different } \\
\text { type of walk }\end{array}$ & 1 & $\begin{array}{c}\text { normal straight, } \\
\text { circuitous, } \\
\text { multidirection with } \\
\text { start\&stop, distorted } \\
\text { speed } \\
\end{array}$ \\
\hline$[15]$ & 2015 & One Plus One & $\mathrm{Be}$ & 26 & 10 steps & 3 & 1 & - \\
\hline [16] & 2016 & One Plus One & $\mathrm{Be}$ & $\begin{array}{c}30 \\
(22 \mathrm{M}-8 \mathrm{~F}) \\
\text { age }[16,65]\end{array}$ & 10 steps & 3 & 1 & $\begin{array}{l}\text { The experiments are } \\
\text { also carried out on } \\
\text { ZJU-gaitacc and } \\
\text { OU-ISIR Inertial Sensor }\end{array}$ \\
\hline [28] & 2016 & $\begin{array}{c}\text { Asus Zenfone 2, } \\
\text { Samsung S3 Neo, } \\
\text { Samsung S4, LG } \\
\text { G2, LG G4, Google } \\
\text { Nexus } 5\end{array}$ & F.R.TP & 50 & $\begin{array}{l}\text { not } \\
\text { reported }\end{array}$ & variable & $\begin{array}{l}\text { variable } \\
\text { (sessions } \\
\text { last about } \\
5 \mathrm{~min})\end{array}$ & - \\
\hline$[38]$ & 2017 & \multicolumn{7}{|c|}{ ZJU-gaitacc dataset } \\
\hline$[52]$ & 2010 & not reported & $\mathrm{TP}$ & 36 & variable & variable & 1 & $\begin{array}{l}\text { Walking, Jogging, Stairs } \\
\text { Up, Stair Down }\end{array}$ \\
\hline$[69]$ & 2011 & \multirow{2}{*}{$\begin{array}{c}\text { Google G1 } \\
\text { (AK8976A) 50Hz }\end{array}$} & \multirow{2}{*}{ R.H. } & \multirow{2}{*}{$\begin{array}{c}48 \\
(38 \mathrm{M}-10 \mathrm{~F})\end{array}$} & \multirow{2}{*}{$37 m+37 m$} & \multirow{2}{*}{$\begin{array}{l}2 \text { (from the } \\
\text { same walk) }\end{array}$} & \multirow{2}{*}{2} & \multirow{2}{*}{$\begin{array}{c}\text { 26.5s per walk } 1108 \\
\text { samples (less then } 42 \mathrm{~Hz} \text { ) }\end{array}$} \\
\hline$[68]$ & 2011 & & & & & & & \\
\hline$[71]$ & 2012 & $\begin{array}{c}\text { Motorola } \\
\text { Milestone } 100 \mathrm{~Hz}\end{array}$ & R.H. & $\begin{array}{c}36 \\
(29 \mathrm{M}-7 \mathrm{~F})\end{array}$ & $\begin{array}{l}\text { about } \\
15 \mathrm{~min}\end{array}$ & $\begin{array}{l}12 \text { normal } \\
\text { pace } 16 \text { fast } \\
\text { pace } \\
12 \text { normal } \\
\text { pace } \\
\end{array}$ & $\begin{array}{l}2 \text { (24 days } \\
\text { delay) }\end{array}$ & - \\
\hline$[54]$ & 2014 & $\begin{array}{c}\text { Samsung Galaxy } \\
\text { S3, Samsung } \\
\text { Galaxy S4, Google } \\
\text { Nexus 5, Intel Xolo }\end{array}$ & variable & $\begin{array}{l}3 \text { different } \\
\text { datasets }\end{array}$ & variable & variable & variable & $\begin{array}{l}\text { The } 3 \text { datasets are used } \\
\text { for different tasks }\end{array}$ \\
\hline
\end{tabular}

The datasets before the double line are used in works relying on step/cycle segmentation, while the others are used in works that process fragments of signals. In the Device Position column, $\mathrm{B}=$ back, $\mathrm{F}=$ front, $\mathrm{R}=$ right, $\mathrm{L}=\mathrm{left}, \mathrm{C}=\mathrm{center}$, $\mathrm{Ar}=$ arm, $\mathrm{A}=$ ankle, $\mathrm{Be}=$ belt, $\mathrm{H}=$ hip, $\mathrm{W}=$ waist, $\mathrm{Wr}=$ wrist, $\mathrm{TP}=$ trousers pocket, $\mathrm{T}$ =thigh. 


\subsection{An Alternative to Smartphones: Smartwatches}

The preceding literature review shows that several studies on gait recognition based on wearable sensors exploit smartphones, since accelerometers and gyroscopes are practically ubiquitously embedded in these devices. This allows to exploit them for free. Smartwatches are an apparently promising alternative, yet not significantly explored till now. They are included in this section, because, if used normally, the captured signals are affected by a variety of factors extraneous to gait kinematics itself. Arm movements, either voluntary or involuntary, create an acceleration effect that overlaps, if not completely cancels, the one produced by walking. Due to the nature of the captured data, works in literature started using these devices mostly for activity detection and recognition. One of the first (hypothesized) uses of smartwatches in addition to body-worn sensors for activity recognition is described in Reference [84]. The extension to gait recognition is more recent.

The work in Reference [11] does not exploit a smartwatch, but it is interesting to discuss it in this context, because it adopts a kind of processing that can be extended to such devices. The experimental set-up includes a pair of Shimmer3 IMU sensors, one positioned on the right tight (like a smartphone) and one positioned on the left wrist (like a smartwatch). The captured signals are processed and classified independently: only the comparison of the achieved performance is reported, with no attempt for fusion. The dataset is collected from 15 subjects, walking six times along a hallway, twice at their normal preferred pace, twice at fast pace and twice with hands in their pockets. Finally, subjects are asked to perform random gestures to simulate false cycles. Capture appears to happen in a single session. The first step of the common workflow entails walking detection, through peaks of the magnitude vectors. It is interesting to point out that, while the general trend of the two signals is very similar, the wrist one has a much lower amplitude. Due to the use of the same kind of sensor in both positions, this is probably due to the attenuation of the acceleration produced by gait when the accelerometer is farther from feet or from a body part directly involved in the movement, especially if the arms do not swing. As a consequence, the threshold used for magnitude peaks detection is lower. Afterward, since the wrist movement is more irregular than the movement of legs, an autocorrelation-based filter is used for the wrist signal to discard too irregular cycles. The following processing is the same for the data from both sensors. A Butterworth filter at $20 \mathrm{~Hz}$ is applied and three additional vectors are computed for each cycle besides those provided by the accelerometers on the $\mathrm{x}, \mathrm{y}$, and $\mathrm{z}$ axes, namely, magnitude and horizontal and vertical components computed as in Reference [54]. The six vectors are used in different combinations for statistical feature extraction (max, mean, median, standard deviation, skewness, kurtosis, autocorrelation of feature samples, etc.). Common features are added, e.g., signal length, to compose the final feature vector. Three approaches are compared. In the first one, data from the pocket sensor is used, considering magnitude, horizontal, and vertical components (mhv). In the second and third approach, data from the wrist sensor are used, considering either magnitude, horizontal and vertical components (mhv), or $\mathrm{x}, \mathrm{y}, \mathrm{z}$ axes and magnitude (xyzm), respectively. During verification (against the owner of the device), the same procedure is carried out and an anomaly score is assigned to each probe cycle with respect to subject's gallery cycles using Euclidean distance and Nearest Neighbor. A first part of the results reports a comparison between walking detection from data from the two positions, showing that the algorithm (with the necessary described modification) produces a similar number of detected cycles, sufficient for subject recognition. This is also due to the autocorrelation filter applied to the wrist signal that is able to discard all the random hand movements. Subject verification is both evaluated by AUC from the ROC curve and EER. As for the pocket sensor, the system achieves AUC $=99.6 \%$ and EER $=2.5 \%$. The interesting information comes from comparing the results from the two approaches using the 
wrist sensor. For mhv the results are AUC $=97.3 \%$ (only slightly lower than pocket), but EER $=$ $8 \%$, with a dramatic worsening. On the contrary, for xyzm AUC $=99.6 \%$ (overall, user verification is scarcely affected either by sensor position or by the choice of feature axes) but EER $=2.9 \%$, therefore returning to values comparable to the pocket setting. This seems to demonstrate that information from the wrist sensors is more effectively exploited using data from $\mathrm{x}, \mathrm{y}, \mathrm{z}$ axes and magnitude. The results are improved by applying the auto-correlation filter, with a higher impact on wrist data. Identification is also assessed, to take into account a scenario where different subjects can wear the sensor from time to time. To this aim, 5 classifiers are compared, namely, 1-NN, Multilayer Perceptron, Random Forest, Rotation Forest, and Multinomial Logistic Regression. The best result is achieved by $1-\mathrm{NN}$ with the pocket sensor, while on the average pocket $(97.3 \%)$ and wrist with xyzm (97.4\%) are almost identical.

Reference [48] proposes to pair a smartphone and an LG G smartwatch on the non-dominant hand. This choice allows less noise created by common actions usually carried out by the dominant one. Data are captured from both accelerometer and gyroscope of the smartwatch, while the smartphone is exclusively used to trigger data collection, receive data and retransmit them to the server for recognition. A dataset is collected from 59 subjects during a $5 \mathrm{~min}$ walk. The overall approach, both the strategy for signal segmentation and the number and type of features extracted, are the same used for smartphone [52]. The classifiers tested in this case are Multilayer Perceptron (MLP), Random Forest, Rotation Forest, and Naive Bayes. A 59-class training is carried out for experiments in identification modality. As for verification/authentication, each user has an own authentication model, with half data used for training and half for testing. Results are mostly reported on a per-user basis, but in general results provided by accelerometers data are better than gyroscope ones.

Reference [98] uses a real smartwatch (a Samsung Gear Live Smart Watch) for implementing the Gait-watch proposed system. The authors propose a kind of context-aware gait-based user recognition, that takes advantage of preliminary activity detection. Training dictionaries are collected for the different actions taken into account, so that identification is performed on the corresponding dictionary. An activity dataset from 15 subjects is collected to train the system for the preliminary action recognition. The seven different activities include three with arm swing (normal walk, walk upstairs, and walk downstairs) and four without (walking while writing text on the phone, walking while calling with the phone, walk with hand in the jacket pocket, and walk with hand in pant pocket). Each subject is asked to walk for $3 \mathrm{~min}$ for each activity, for a total of about $5 \mathrm{~h}$ of training data. Gait signals are segmented in cycles for training purposes, and dictionary creation is improved by a projection optimization algorithm [87]. The gait dataset used for subject recognition includes 20 volunteers in two sessions of data acquisition separated by a week. The terrain includes plain, grass and asphalt (but slope are not mentioned) and subjects' dresses and shoes may change across sessions. Subjects perform the considered activities in equal proportion, for a total of $10 \mathrm{~h}$ of gait data. A 10 -fold cross-validation is carried out. During testing, the first processing step is walking detection. The best classification accuracy is achieved by adopting a K-NN classifier with $\mathrm{K}=3$ and a window size of $2 \mathrm{~s}(98.6 \%)$. After activity recognition, gait cycle segmentation is carried out. The raw accelerometer signals along the three axes fluctuate in a significant manner due to arbitrary body movements. However, a kind of regular pattern is produced by gait along the gravity direction, so that gravity coordinates can be used, obtained applying the rotation (R) matrix provided by Android to transform acceleration data over $\mathrm{x}, \mathrm{y}$, and $\mathrm{z}$ axes into $\mathrm{E}$ (st), $\mathrm{N}$ (orth) and $\mathrm{G}$ (ravity). After applying a Butterworth filter, gravity-related peaks allow walking detection using the approach in Reference [81]. A sparse fusion method combines information from different gait cycles. Subject recognition is carried out both without and with preliminary action recognition (after a corresponding kind of training), with an improvement of up to $20 \%$ of Recognition 
Accuracy (the rate of correct classifications) with respect to the so defined conventional method that uses a common pool of undifferentiated gait cycles to train subject recognition.

Regarding the best choice of features to exploit for smartwatch-based gait recognition, the experiments in Reference [2] explore both time-related and frequency features. Moreover, both accelerometer and gyroscope signals are captured. The analysis is carried out on a dataset of 36 subjects, with gait captured during six sessions of about 2 min each at normal speed on different days. Besides walking, the subjects also carry out actions like opening a door and turn. The reported analysis compares data for single users, taking into account elements like same/different session and the number of features exploited. The proposed solution is a dynamic feature vector for each user.

\subsection{Device Orientation and Phase Changes}

One of the possible problems in gait recognition via wearable sensors is related to the orientation of the acquisition device, that can cause very different signals even for the same person. The simplest solution (Section 5) exploits the magnitude vector instead of the three separated axes, but the correlation among the axes values is lost. Other kinds of solutions are generally more complicated and involve geometric transformations of a time series within a 3D space.

The approach in Reference [102] computes orientation invariants for both accelerometer and gyroscope data and exploits them to extract features that characterize locomotion dynamics and are robust to sensor orientation. Given an acceleration (rotation) time series with fixed time intervals, a two-dimensional matrix (Gait Dynamics Image) captures invariant dynamics over time and interactions within each cycle. Matrices are compared after extracting i-vectors, similarly to speech processing.

Signal correspondence is computed in Reference [67] using cyclic dynamic programming and relative sensor-orientation estimation. Even here the magnitude vector is exploited as a rotationinvariant feature.

Phase registration is a possible problem that may affect gait signal matching, especially with unsegmented signals. The work in Reference [92] proposes to exploit a linearized Time Warping Function (TWF) on the gallery samples to normalize signals and improve matching accuracy.

Comparisons are mostly carried out on OU-ISIR and show a significant decrease in the EER. For other proposals, see Section 7.1 (smartwatches).

\subsection{Cross-Device Setting}

There are few works in literature facing the problem of cross-device setting. In a real scenario, especially in identification settings, e.g., to grant access to authorized people without an explicit authentication, it is possible to need to recognize a subject even if using a different smartphone from the enrollment one. In general, cross-device signal matching is expected to achieve lower accuracy, unless using a preprocessing procedure that tries to realign the signals in terms of recorded values. As discussed in Section 3, even two identical accelerometers are likely to produce different values in identical positions. Consequences are negligible for, e.g., operations related to screen rotation or gaming, but not when the signal is acquired for biometrics purposes. To solve this problem, Reference [13] proposes a high-level procedure that does not require to act on the sensor itself. It collects sensor data for ten seconds in the six positions where it is possible to know exactly the ideal/expected acceleration values on the three axes (see Figure 3(b)). After this acquisition phase, data are averaged and the procedure computes the distance from the expected value for each axis. These deviations are stored on the device and taken into account during future acquisitions. This procedure requires only about $2 \mathrm{~min}$, it is one-shot, and it provides a file that can be also exploited by all the other applications running on the device to normalize the accelerometer data. It is 
possible to apply it even after the data acquisition. The performances achieved after this preprocessing seem to overcome those obtained using the original signal, especially in cross-device settings, as expected (up to $225 \%$ improvement in closed set identification), but also when comparing the data from the same device, though at a lower extent (up to $216.7 \%$ improvement in verification).

\section{LESS INVESTIGATED TOPICS}

The works in this section address very specific issues, so that it is worth describing them separately.

\subsection{The Effect of Gait Stabilization}

The work in Reference [26] investigates the correlation between the recognition results and either the position in the walk sequence or the number of cycles used for matching. The two parameters are also considered together. The authors exploit the same techniques in Reference [25] for preprocessing (plus noise reduction by Low Pass Filter), cycle segmentation, and recognition. The walk cycles are divided into three groups (Beginning, Middle, and End). After that, the system chooses the $k$ most representative cycles by DTW (those that achieve the lowest average distance with all the others). This is the most computationally demanding part but it is independent of $k$, which is changed from time to time ranging from 1 to the total number of cycles. This procedure is repeated and accuracy is computed both for the entire walk and for each of the three groups of cycles. The results show that a higher $k$ has a positive impact on recognition, but the gain is not that significant, while the matching computational time is directly proportional to $k$. As an interesting outcome, the cycles in the End group provide better results with respect to the other two groups, even though lower than those from the entire walk. This is probably due to gait stabilization, i.e., to a tendency to better regularity after some cycles. Therefore, it seems possible to reduce computational time by taking into account only the last representative cycles, with just a little accuracy decrease.

The experiments in Reference [60] confirm the above results. The segmentation procedure is slightly different from Reference [16]: the parameter stepequilibrium (see Section 6.1.3) is computed as $\mu-\sigma$ ( $\mu$ is the mean and $\sigma$ is the standard deviation of signal values), and the segmentation parameters are computed also for probe samples instead of applying a best-fit strategy. The dataset used for the experiments is ZJU-gaitacc, which is large enough to reliably generalize the results. The gait signals are segmented into steps and comparisons consider only the last two, three, four, and five steps, respectively. The results show that two steps are sufficient for acceptable verification and identification performance, given that they are taken from the last part of the walk signal. On the contrary, the first steps achieve poor results. As a side consideration, this seems to testify that long walks are more reliable than short ones.

\subsection{Optimizations for Gait Recognition Systems}

State-of-the-art optimization techniques for gait recognition via wearable sensors that are presented here do not only regard the computational demand but also the problems that it can rise or worsen. As for any other kind of mobile recognition system, the problem of energy consumption is not negligible and must be taken into account in the design of a real system. It is to notice that the strategies in the previous subsection can also be used to reduce the computation/energy demand of comparisons, since they exploit a subset of samples of the original signals.

The proposal in Reference [63] presents a possible strategy to reduce energy consumption during continuous gait authentication (see also Section 9). At present, the acquisition of accelerometer signals by any kind of mobile device requires the use of CPU activation and processing, causing a power consumption greatly impacting the battery life. The situation taken into account entails acquiring and processing accelerometer data, even when the subject is not moving. The authors 
suggest exploiting a pedometer sensor, i.e., a low power consumption sensor able to detect device movement without passing through CPU activation. This allows triggering the recording phase only when needed. However, the pedometer is not a standard sensor in present mobile devices.

Another strategy to reduce energy consumption is presented in Reference [17]. The considered scenario entails an event-triggered acquisition. In this case, the problem addressed is the possible continuous background execution of the authentication app, since the goal of the proposed system is, for example, to automatically open a door at the end of a hallway only for authorized persons. The described solution includes an external source (a Bluetooth 4.0 beacon) that continuously sends its ID. The designed mobile app remains idle until the Bluetooth interface receives a specific ID and generates a wake-up event. In practice, when the mobile phone is within the transmission range, if the received ID corresponds to a beacon registered as a start element, the app automatically starts the data acquisition. When the smartphone later reaches a beacon corresponding to a stop event, it stops data acquisition and sends the data to the recognition server. The advantages of the use of beacons are twofold. Bluetooth 4.0 has a little impact on energy consumption and, in any case, it could be required by other applications too. Moreover, a beacon has a battery with an estimated lifetime of about 2 years even working constantly. Such a lifetime can be further increased using a shutdown procedure for the possible day periods when the system is not required to work.

\subsection{Gender Recognition by Gait}

It could be interesting to investigate gender recognition by wearable sensor data. However, as already mentioned in Section 4, the largest publicly available datasets do not provide any gender information, so that addressing this research topic first requires collecting new datasets. At the best of our knowledge, a single work [46] has tackled the problem so far. Walking data from both the accelerometer and the gyroscope are captured from 109 subjects (46 with a Samsung Galaxy S-II GT-I9100 and 63 with a Note-II N7100, with about the same number of males and females). The subjects are asked to keep the smartphone in one of the frontal trouser pockets and to walk twice at three different speeds (slow, normal, and fast) for a total of 654 walking signals. The gait signals are normalized both in amplitude (by z-mean normalization) and in time (re-sampling at $100 \mathrm{~Hz}$ by cubic spline interpolation), and denoised by moving average. Minima in the z-axis are used to identify cycles, refining their positions by the data from $\mathrm{x}$ and $\mathrm{y}$ axes. Histograms of Oriented Gradients (HOG) features [12] are exploited, which are generally used in image processing. The gradients are computed using different masks and then a 6-bin histogram is computed. A bootstrap aggregation is exploited for classification and regression. In this ensemble method, multiple predictors are aggregated, in this case, an ensemble of decision trees. Bagging generates an aggregated model by combining the outputs of the individual models. Classification uses plurality voting. The accuracy of this strategy for gender recognition ranges from about $70 \%$ to about $94 \%$ depending on the test scenario (e.g., single speed or cross-speed, and using accelerometer, gyroscope, or both).

\subsection{Wearable Sensors Capturing Different Physical Measures Exploited in Gait Analysis}

The study in Reference [47] proposes a myography sensor to detect and extract gait cycles. Experiments consider the four gait phases starting with Initial Contact, Mid Stance, Pre-Swing, and Swing (see Figure 1). Data are acquired by a force myography ankle band (an array of eight forcesensing resistors), and a high-speed camera is used for ground-truth labeling. The nine healthy volunteers involved in the acquisition (five males and four females) are asked to walk on a treadmill at three different speeds $(1,1.5$, and $2 \mathrm{~km} / \mathrm{h})$. After a $2 \mathrm{~min}$ trial, followed by a rest of $2 \mathrm{~min}$, each subject walks for 1 minute at each speed. This second part is repeated five times with $2 \mathrm{~min}$ breaks. 
A total of 14 features are extracted using a sliding window of $125 \mathrm{~ms}$ with an overlap of $93 \mathrm{~ms}$, i.e., root-mean-square, sum of absolute values, mean absolute deviation, and others. The features are normalized for matching purposes. Linear Discriminant Analysis (LDA) is exploited for the data points classification. The gait phases are used as classes. Two independent observers label the start and the stop of the phases used for the training set according to the video sources. The testing phase determines the phase for each data point. Ad hoc rules detect suspect phase transitions (not compatible with a normal walk) to discard them. A cross-trial validation method is used to evaluate the accuracy of walking phase identification: one of the five trials per user at each walking speed is chosen in turn as the testing data and the remaining ones at the same speed are assigned to the training set. The reported overall accuracy ranges from $89.5 \% \pm 2.5 \%$ to $92.9 \% \pm 1.2 \%$, depending on speed, for an average accuracy of $91.3 \% \pm 3.3 \%$. The slowest the walk, the less accurate the phase classification. Results could be used to segment the signals from the accelerometer, but this approach requires a non-conventional set of sensors and sensor synchronization. No subject recognition attempt is reported.

The method in Reference [61] exploits gait data by pressure sensors put in a shoe sole. The dataset used had been collected to test an approach to diagnostic gait analysis presented in Reference [42]. It consists of 50 pressure signal samples recorded by 10 subjects in an ambulatory. For verification, a first method exploits Euclidean Distance between pairs of samples. The best reported result is a FRR of $7.2 \%$ with a FAR of $0.4 \%$. A second method exploits a Gaussian Mixture Model (GMM) for each user, computed during the enrollment. Each sample is considered as a feature vector. GMM training is done by expectation maximization (EM). During verification, the system automatically updates the individual GMM model and the acceptance threshold(s) according to the new information. The new experiments report $80 \%$ of positive identification rate and $86 \%$ of impostors rejection rate.

The acquisition technique in Reference [75] relies on plantar pressure imaging (PPI), capturing the way each individual interacts with the ground, within common stereotypical constraints: heel strike, roll to the forefoot, then push-off with the distal forefoot and toes. This cyclic process takes about $0.7 \mathrm{~s}$ when walking at normal speeds (about $1.2 \mathrm{~ms}^{-1}$ ). PPI systems can be included among floor-sensor-based approaches, and typically use an array of hundreds or thousands of pressuresensitive sensors. Spatial and temporal resolutions are in the order of $5 \mathrm{~mm}$ and $100 \mathrm{~Hz}$, respectively. These measures are rendered in the form of images, where the foot can be easily isolated from the environment using low-pressure thresholding, and that can be easily aligned. PPI-based biometric identification had already been proposed, but with very small datasets (a maximum of 30 subjects in Reference [90]). The purposes of this study are thus to explore the feasibility of PPI-based gait recognition on a larger sample of subjects (104), and to systematically compare a variety of spatial alignment procedures, and of features and feature extraction procedures. Computer vision-based processing and classification are applied to PPI images and, therefore, we do not add further detail on this approach. It would not be suitable for this survey if the weight-based approach had not been recently made "wearable" by the work in Reference [100]. The latter is worth mentioning just because of the novel strategy to gait recognition, though somehow related to the above one, that requires eight weight sensors to be embedded directly in the shoe insole. It seems that values from the sensors are mapped onto corresponding time series and processed as such. Weight is used as a feature together with the way of walking (time series of weight distributions produced while walking). A very small dataset is used for testing, with apparently 14 gallery and 14 probe samples, a pair per user. No indication is given about the length and conditions of the walk. Both a Gaussian Radial Basis Function (GRBF) SVM and a Naive Bayes (NB) Classifier are tested, with an average verification accuracy of $83.88 \%$ for NB and $99.60 \%$ for the SVM-GRBF. The problem is represented by false acceptances. Even if the results are obtained over a small dataset, this interesting capture 
modality would solve the problem of device orientation and position. However, specially equipped shoes are needed (not such unrealistic compared with a band of sensors all around the body).

\subsection{Multibiometric Systems Including Wearable Sensor-based Gait Recognition}

The following two works exploit the synchronization of video and inertial data for gait recognition.

The proposal in Reference [91] presents Proprio-Extero Matching IDentification (PEM-ID). Data are acquired by a $640 \times 480$ camera at $30 \mathrm{fps}$ and by the SparkFun 6 DoF IMU board, with a MMA7260Q accelerometer, embedded into a small computer on the person's body. A motion signature consists of landmark features extracted from cameras and accelerometers. Data from both camera and accelerometer are acquired from two subjects in 12 walks of about 10 steps; beyond this distance, for sake of image quality, only the accelerometer is used. The feature vector includes the timestamps of the heel-strike and midswing events for both video and acceleration data. For the video sequence, heel-strike and midswing events are characterized by feet at their farthest or closest distance, respectively. Regarding the accelerometer signal, it is known that during the gait cycle, the vertical acceleration of the body's center of mass reaches its maximum at the midswing and its minimum at the heel-strike. In a first set of experiments, a single person at a time walks across the camera field of view (FOV) for 12 runs, changing the source and destination points. This is repeated for two different people (24 walk sequences). Permutations of the experimental data allow simulating multiple-person scenarios. The matching exploits a suitable distance metric. In a second set of experiments, three people cross the camera FOV, for eight experimental runs, each time entering/leaving from different directions. The PEM-ID system identifies the only person in the scene wearing an accelerometer. The recognition procedure searches the best match that globally minimizes the distance between video and accelerometer features. The average recognition rate is $87.5 \%$.

The system in Reference [103] combines RGBD (RGB plus depth information) and accelerometer data. The acceleration signals are collected by an Android smartphone (of an unspecified model) in a front pocket. The video sequence is collected by a Kinect 2.0 at $0.5 \mathrm{~m}$ height. The RGB and the depth stream are combined into a single VGA stream (8 bits per pixel). A group of acceleration data is the sequence of acceleration values from the walk between the two ends of the hallway. Three different datasets are collected. The first one includes data from 10 subjects with 100 groups of acceleration data and RGBD data each, half at normal pace and half at fast pace along an $18 \mathrm{~m}$ hallway. The data from the accelerometer are collected, divided into steps and interpolated at $50 \mathrm{~Hz}$. The video data are captured asking the subjects to walk 100 times 1 to $5 \mathrm{~m}$ away from the Kinect. Video and smartphone data are not synchronized. The second and the third datasets contain data from 50 subjects with synchronized video and accelerometer data. The third dataset contains more acquisitions in different conditions (e.g., arms position). For accelerometer signals, events are midswings and heel-strikes, identified as above. Separate features are extracted from the accelerometer (magnitude vector) and video data. For accelerometer data, the system applies Principal Component Analysis to the gait curves exploited as training vectors; then the system uses the computed basis (EigenGaits) to map a new gait signal onto the derived space. For the video, the system uses Dense 3D Trajectories-Based Gait Representation and exploits a bag-of-words strategy to encode and then cluster the extracted trajectories using the k-means algorithm. The recognition exploits the concatenation of the features extracted from accelerometer data and from the video: this vector is used to train a SVM classifier (using Pearson's correlation coefficient as a measure for signal similarity). SVM reaches an accuracy above $90 \%$, with peaks of $96 \%$, in different experimental scenarios.

To the best of our knowledge, a single work so far has fused wearable sensor-based gait and voice recognition to improve their accuracy [95]. Recognizing users by gait is possible when they 
carry mobile devices while walking, and also recognizing a speaker is also a viable operation while talking via the mobile device or close to it. The performance of the combined approach is demonstrated to be significantly better. Experiments are carried out using voice samples and gait acceleration data from 31 subjects (19 males and 12 females) participating in two different sessions. The three-dimensional accelerometer module includes two perpendicularly positioned Analog Devices ADXL202JQ accelerometers and a laptop computer equipped with National Instruments DAQ 1200 card. The accelerometer signals are recorded at $256 \mathrm{~Hz}$. During each session, the subjects walk along an about $20 \mathrm{~m}$ corridor with normal, fast, and finally slow walking speed, with each of three different placements of the accelerometer module: hip pocket, chest pocket, and suitcase handle. The accelerometer module does not freely move inside the pockets, but pockets are attached to mock-ups of clothes and their shifting leads to differences in accelerometer positioning during data collection for training and testing. The speech database used for experiments contains five utterances per speaker, each being an eight-digit string pronunciation. The four first utterances are collected during a first session and used for training. The fifth speech sample is recorded during a second session and is used for testing. The speech samples (at $8,000 \mathrm{~Hz}$ ) are collected in a quiet environment. They are then normalized and contaminated with white, pink, city and car noise with three SNR conditions, 20,10, and $0 \mathrm{~dB}$. Pink and white noise are artificially generated. City and car noise are taken from NTT-AT Ambient Noise Database. ${ }^{5}$ Gait processing and recognition are carried out by the already discussed methods in References [1,59], entailing both a score determined by the correlation of gait segments and one computed via FFT (see Section 6.2). The speaker recognition exploits the text-independent MASV (Munich Automatic Speaker Verification) speaker verification environment. ${ }^{6}$ The fusion of gait and voice similarity scores is carried out by Weighted Sum fusion, after normalizing scores of each modality to the range $[0,1]$, with the weights derived from the expected reliability of each modality, estimated in advance from the achieved error rates. The best performance using separately the traits is EER $=14.1 \%$ for gait recognition with correlation with the accelerometer module in hip pocket, EER $=13.7 \%$ using FFT with the accelerometer module in chest pocket, and EER $=2.82 \%$ for speaker recognition with $20 \mathrm{~dB}$ SNR with city noise. The best result with fusion is EER $=1.97 \%$ with city noise $20 \mathrm{~dB}$ and the accelerometer module in the chest pocket.

\section{ROBUSTNESS TO IMPERSONATION ATTACKS}

The problem of spoofing/impersonation is relevant in biometrics. In the case of gait patterns acquired by wearable sensors, it is interesting to know at which extent a spoofing/impersonation attack might be successful. Different specific scenarios have been analyzed.

The experiments in Reference [36] also include the evaluation of minimal effort mimicking for gait biometrics. The datasets used for this work and for the following ones by the same group are collected using a Motion Recording (MR) sensor. The magnitude vector is computed from the acquired signals, linearly interpolated and denoised by moving average filter. Cycle detection exploits local minima, starting from the first group of 250 samples and considering that a cycle contains about 100 samples. Cycles are normalized in time to have exactly 100 samples each. An average cycle is computed for each walk by taking the median value of corresponding samples in walk cycles. Euclidean distance between average cycles provides the matching score. In the kind of attack analyzed in this work, impostors have only a limited number of attempts and can spend a short time to study the target. It is not a zero-effort attack but entails very low engagement. The results are

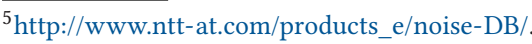

${ }^{6} \mathrm{http}: / /$ www.bas.uni-muenchen.de/Bas/SV/.
} 
quite optimistic about the robustness of gait against this kind of attempt, but experiments involve a limited number of samples.

The experiments in Reference [34] are more specifically focused on spoofing attacks, and exploit a larger dataset of 100 subjects. The work investigates two types of attack scenarios. The first one is similar to the preceding work, dealing with a minimal effort impersonation. In the more complex scenario, each attacker knows who is the person in the dataset with the most similar gait. The results confirm that a minimal effort impersonation attack on gait does not significantly increase the chances of impostors being accepted, but they also demonstrate that an attacker with knowledge of the closest match in the database can represent a serious threat to the gait authentication system.

The study in Reference [29] demonstrates that knowing the gender of the spoofed target helps the attacker. One point in the final discussion in Reference [34] is whether training can improve attackers' performance.

The conclusions reached by different works are not univocal. More recent experiments in Reference [63] take also some amount of attacker training into account. The work exploits energy consumption reduction (Section 8.2), since the experiments hypothesize continuous authentication to verify the identity of the owner of the mobile device. Preprocessing applies mean subtraction for accelerometer signal denoising and then the system computes the magnitude vector. The magnitude vector undergoes linear interpolation and filtering by Savitzhy-Golay algorithm. The cycle length $l$ is estimated and cycles are extracted exploiting local minima at approximately $l$ samples distance. Outlier cycles are computed by DTW and removed. Recognition is based on majority voting over DTW pairwise comparisons between cycles. A user is recognized if at least $50 \%$ of such comparisons pass the (fixed) voting threshold. Two datasets are used for different experiments: zero-effort, minimal effort (reenact), and coincide. In both cases, data are collected by one Sony Xperia Z3 and two Sony Xperia Z5-Compact smartphones. The first dataset collects data from 35 subjects along a $68 \mathrm{~m}$ hallway. It is used in all-against-all matching, which simulates in some way zero-effort attacks (simply a false identity is claimed). The system achieves an EER of $13 \%$. The second set of experiments is specifically designed to simulate impersonation attacks. It involves a group of five attackers, that are all actors trained and specialized to mimic body language and motions. The four victims are normal users that have similar physical characteristics to those of at least one of the attackers (one of the victims is suitable for two attackers). During the enrollment, a second dataset is collected asking the victims to complete two rounds of a $16 \mathrm{~m}$ corridor at a normal speed (3-4min), while the respective attacker observes the victim's phone placement and walking style. Afterward, the attackers are allowed to further observe the respective victims also noting the body movements and other characteristics useful for imitation. Then, an attempt to be recognized as the victim is carried out. No attacker reaches the requested amount of votes to be accepted. The last scenario (coincide) is specifically designed to analyze whether synchronization of steps and other body movements can help attackers to learn and reproduce a victim's gait. A victim and its attacker walk side by side at the victim's normal pace. The rationale for this is that it seems that people walking side by side normally tend to adapt to each other's walking pace, which could make the attack more effective. The victim's and attacker's smartphones are connected to each other over a Bluetooth channel, and the gait authentication application runs in verification mode on both smartphones for $15 \mathrm{~min}$. Live templates from the victim's smartphone are transferred in real-time to the attacker's smartphone and compared against the attacker's live templates. Notwithstanding the more favorable condition, no impostor reaches the needed confidence score to be accepted. Even if a few subjects are involved, considering that attackers are well trained in impersonating behaviors, these results seem to prove the robustness of gait to impersonation attacks. Another interesting observation is that attackers' imitation capability generally decreases along the walk. 
To the best of our knowledge, a single work has investigated spoofing of a silhouette-based gait biometric systems [40]. Experiments testify that it is possible, though not as easily as for face. The latter can be easily achieved by a photograph of the target person's face, whereas gait spoofing may require greater efforts, e.g., to select a target with a similar body build to the attacker, imitate the clothing and mimicking the walking style. Given the possibility to combine video and wearable sensor data (see Section 8.5 above), it would be interesting to measure the possible increase of robustness provided by simply combining gait recognition by the two different modalities.

\section{SUMMARY AND CONCLUSION}

As pointed out in Section 6.5, most approaches exploit gait biometrics for verification. The owner of the device, e.g., a smartphone, enrolls as the only subject in the system gallery, and this represents an implicit claim of identity. Therefore, the application only matches the corresponding template with the probe acquired from time to time. On the contrary, in computer vision-based approaches, which are mostly used in video surveillance, there is no possibility for identity claim; biometric sample acquisition might also be covert or the user might not be (continuously) aware of it. The aim, in this case, is to identify an unclaimed identity, to match it against an either white (allow transit) or black (raise an alarm) list, or to continuously re-identify (different) subjects in videos. However, it is also possible to hypothesize a wider use of wearable sensors-based approaches. Low-consumption radio devices might trigger at a distance the capture of the gait signal of an approaching subject. The signal might be sent to a server controlling the access to a protected area, that might automatically open a secured door to authorized subjects. The kind of interaction entails a mixture of awareness (the user has to voluntarily install a suitable application) and unaware capture (the user has nothing to explicitly do so that the capture process can be maintained transparent). Moreover, for sake of defenders of privacy issues, it is to say that, while face and silhouette can be acquired in a completely covert way, this is not possible for the accelerometer signal, unless using spy story techniques (an accelerometer hidden on an unaware subject, or injection of a hidden capture application) or modifying the production chain of, e.g., a smartphone to set up a default/on-demand remote transmission of the accelerometer signal not necessarily triggered by the user. On the other hand, especially when transmitting the complete accelerometer signal, and when storing it remotely, it is worth applying some encryption technique to protect it from steal/spoof. Actually, this kind of issue is common to all biometrics-based applications and is an active research area in itself. Regarding recognition accuracy, possible limitations of this biometric trait can be compensated for by using a multibiometric approach, by adding, e.g., face recognition from images acquired by a camera at a reasonable distance and with a frontal perspective on the person approaching a secure gate. This would be a common setting, after all, requiring no special behavior from the user, but walking normally: an average walking pace is highly probable while entering a protected zone.

It is worth devoting a special discussion to the use of the accelerometers and other sensors embedded in smartwatches. According to the works mentioned in Section 7.1, this kind of sensors is suitable to distinguish walking state from standing, and for activity detection and possible recognition. However, the signal from the smartwatch accelerometer presents some disadvantages for gait recognition. Contrarily to the smartphone, the watch is positioned in a fixed position and does not change its orientation relative to the wrist. However, the orientation of the signal and its distribution over the axes change sharply with (possibly involuntary) arm/hand movements. These get merged with the walk general stereotypic pattern, which may involve arms too but gets disrupted by arm movements. Even the use of the magnitude vector to identify signal peaks, useful for segmentation, is hindered by possible wrist rotations. A reliable walking detection is critical but also a preliminary action recognition can improve the subject recognition. The available datasets 
exploit a controlled capture with a limited number of possible action states, and further studies must investigate the true potential of using these devices that are recently widely spreading.

In conclusion, it is possible to summarize a list of the main open problems: (1) wearable sensorsbased gait recognition suffers from variable device orientation, only partially addressed by aggregate values; (2) variable speed and ongoing actions modify natural gait kinematics as captured by accelerometers and similar sensors; (3) a different ground slope or the kind of shoes, especially with heels can affect long term matching; (4) it is not obvious how to choose the best matching strategy in relation to the walk length, given that the walking pattern stabilizes over a medium-long time; (5) annotated datasets with exhaustive demographic and context variations are not available yet.

The fact that automatic systems could recognize a person from the walking pattern encourages to continue searching for features characterizing this trait. Different approaches attempted in the last years testify the wide range of techniques able to process gait signals and use them for recognition. The performances are generally interesting, notwithstanding the fact that gait is a soft biometrics. Therefore, gait signals can be considered as a good candidate also for supporting hard biometrics. Especially wearable-based signals can be combined with other traits with no interference, since the acquisition device is carried by the user. When combined with gait recorded by other sources (e.g., floor sensors or video), the synchronization of data could benefit all systems involved. When used for identification, a mobile phone or smartwatch itself can be used as a further "ID token," so that the user can be requested to "provide" the right walk to be associated with the correct identity, and also to carry the right device, as for a bank card and its pin code. Another interesting factor is the robustness of gait against impersonation attacks, even by well-trained attackers. This can be a valuable support for a harder biometric trait possibly being less robust to spoofing. As it is happening with other biometrics, especially those based on video/image sources, proposals exploiting CNNs are a new research trend. The major problem with this kind of approach is the present absence of datasets large enough to best exploit the capability of deep architectures. Even with the limitations discussed in this survey, gait recognition is a promising field of research, and can both become a reliable support for hard biometrics, and be used autonomously.

\section{REFERENCES}

[1] Heikki J. Ailisto, Mikko Lindholm, Jani Mantyjarvi, Elena Vildjiounaite, and Satu-Marja Makela. 2005. Identifying people from gait pattern with accelerometers. In Defense and Security. International Society for Optics and Photonics, $7-14$.

[2] Neamah Al-Naffakh. 2017. A comprehensive evaluation of feature selection for gait recognition using smartwatches. Int. 7. Info. Secur. Res. 6, 3 (2017), 691-700.

[3] X. André-Thoma. 1940. Equilibre et équilibration. Editeurs Masson \& Cie, Paris (1940).

[4] Akin Avci, Stephan Bosch, Mihai Marin-Perianu, Raluca Marin-Perianu, and Paul Havinga. 2010. Activity recognition using inertial sensing for healthcare, wellbeing and sports applications: A survey. In Proceedings of the 23rd International Conference on Architecture of Computing Systems (ARCS'10). VDE, 1-10.

[5] Silvio Barra, Maria De Marsico, Michele Nappi, Fabio Narducci, and Daniel Riccio. 2019. A hand-based biometric system in visible light for mobile environments. Info. Sci. 479 (2019), 472-485.

[6] L. Bianchi, D. Angelini, and F. Lacquaniti. 1998. Individual characteristics of human walking mechanics. Pflügers Archiv. 436, 3 (1998), 343-356.

[7] Jorge Blasco, Thomas M. Chen, Juan Tapiador, and Pedro Peris-Lopez. 2016. A survey of wearable biometric recognition systems. ACM Comput. Surveys 49, 3 (2016), 43.

[8] N. Alberto Borghese, L. Bianchi, and F. Lacquaniti. 1996. Kinematic determinants of human locomotion. f. Physiol. 494, 3 (1996), 863-879.

[9] Chen Chen, Roozbeh Jafari, and Nasser Kehtarnavaz. 2017. A survey of depth and inertial sensor fusion for human action recognition. Multimedia Tools Appl. 76, 3 (2017), 4405-4425.

[10] Roger Clarke. 1994. Human identification in information systems: Management challenges and public policy issues. Info. Technol. People 7, 4 (1994), 6-37. 
[11] Guglielmo Cola, Marco Avvenuti, Fabio Musso, and Alessio Vecchio. 2016. Gait-based authentication using a wristworn device. In Proceedings of the 13th International Conference on Mobile and Ubiquitous Systems: Computing, Networking, and Services. ACM, 208-217.

[12] Navneet Dalal and Bill Triggs. 2005. Histograms of oriented gradients for human detection. In Proceedings of the IEEE Computer Society Conference on Computer Vision and Pattern Recognition (CVPR'05), vol. 1. IEEE, 886-893.

[13] Maria De Marsico, Daniele De Pasquale, and Alessio Mecca. 2016. Embedded accelerometer signal normalization for cross-device gait recognition. In Proceedings of the 15th International Conference of the Biometrics Special Interest Group (BIOSIG'16). IEEE, 1-5.

[14] Maria De Marsico, Chiara Galdi, Michele Nappi, and Daniel Riccio. 2014. Firme: Face and iris recognition for mobile engagement. Image Vision Comput. 32, 12 (2014), 1161-1172.

[15] Maria De Marsico and Alessio Mecca. 2015. Biometric walk recognizer. In Proceedings of the International Conference on Image Analysis and Processing. Springer, 19-26.

[16] Maria De Marsico and Alessio Mecca. 2016. Biometric walk recognizer - gait recognition by a single smartphone accelerometer. Multimedia Tools Appl. 76, 4 (2016), 4713-4745. DOI : https://doi.org/10.1007/s11042-016-3654-1

[17] Maria De Marsico and Alessio Mecca. 2017. Gait recognition: The wearable solution. In Human Recognition in Unconstrained Environments: Using Computer Vision, Pattern Recognition and Machine Learning Methods for Biometrics, Maria De Marsico, Michele Nappi, and Hugo Proença (Eds.). Academic Press, 177-195.

[18] Maria De Marsico, Michele Nappi, Fabio Narducci, and Hugo Proença. 2018. Insights into the results of MICHE I-Mobile iris challenge evaluation. Pattern Recogn. 74 (2018), 286-304.

[19] Maria De Marsico, Michele Nappi, and Hugo Proença. 2017. Results from MICHE II-mobile iris challenge evaluation II. Pattern Recogn. Lett. 91 (2017), 3-10.

[20] Mohammad Omar Derawi. 2010. Accelerometer-based gait analysis, a survey. Nor Informasjonssikkerhetskonferanse NISK (2010). Available at http://cv.derawi.com/cv/publications/derawi_nisnet_nisk_gaitsurvey.pdf.

[21] Mohammad O Derawi, Patrick Bours, and Kjetil Holien. 2010. Improved cycle detection for accelerometer-based gait authentication. In Proceedings of the 6th International Conference on Intelligent Information Hiding and Multimedia Signal Processing (IIH-MSP'10). IEEE, 312-317.

[22] Mohammad O. Derawi, Claudia Nickel, Patrick Bours, and Christoph Busch. 2010. Unobtrusive user-authentication on mobile phones using biometric gait recognition. In Proceedings of the 6th International Conference on Intelligent Information Hiding and Multimedia Signal Processing (IIH-MSP'10). IEEE, 306-311.

[23] V. Dietz, J. Quintern, and M. Sillem. 1987. Stumbling reactions in man: Significance of proprioceptive and preprogrammed mechanisms. f. Physiol. 386, 1 (1987), 149-163.

[24] Roger M. Enoka. 1988. Neuromechanical Basis of Kinesiology. ERIC.

[25] Pablo Fernandez-Lopez, Judith Liu-Jimenez, Carlos Sanchez-Redondo, and Raul Sanchez-Reillo. 2016. Gait recognition using smartphone. In Proceedings of the IEEE International Carnahan Conference on Security Technology (ICCST'16). IEEE, 1-7.

[26] Pablo Fernandez-Lopez, Jorge Sanchez-Casanova, Paloma Tirado-Martín, and Judith Liu-Jimenez. 2017. Optimizing resources on smartphone gait recognition. In Proceedings of the IEEE International foint Conference on Biometrics (IFCB’17). IEEE, 31-36.

[27] Dinei Florencio and Cormac Herley. 2007. A large-scale study of web password habits. In Proceedings of the 16th International Conference on World Wide Web. ACM, 657-666.

[28] Matteo Gadaleta and Michele Rossi. 2016. Idnet: Smartphone-based gait recognition with convolutional neural networks. Arxiv Preprint Arxiv:1606.03238 (2016).

[29] Davrondzhon Gafurov. 2007. Security analysis of impostor attempts with respect to gender in gait biometrics. In Proceedings of the 1st IEEE International Conference on Biometrics: Theory, Applications, and Systems (BTAS'07). IEEE, 1-6.

[30] Davrondzhon Gafurov. 2007. A survey of biometric gait recognition: Approaches, security and challenges. In Proceedings of the Annual Norwegian Computer Science Conference. Citeseer, 19-21.

[31] Davrondzhon Gafurov, Kirsi Helkala, and Torkjel Søndrol. 2006. Biometric gait authentication using accelerometer sensor. f. Comput. 1, 7 (2006), 51-59.

[32] Davrondzhon Gafurov, Kirsi Helkala, and Torkjel Søndrol. 2006. Gait recognition using acceleration from MEMS. In Proceedings of the 1st International Conference on Availability, Reliability and Security (ARES'06). IEEE, 6-pp.

[33] Davrondzhon Gafurov and Einar Snekkenes. 2008. Towards understanding the uniqueness of gait biometric. In Proceedings of the 8th IEEE International Conference on Automatic Face \& Gesture Recognition (FG'08). IEEE, 1-8.

[34] Davrondzhon Gafurov, Einar Snekkenes, and Patrick Bours. 2007. Spoof attacks on gait authentication system. IEEE Trans. Info. Forensics Secur. 2, 3 (2007), 491-502.

[35] Davrondzhon Gafurov, Einar Snekkenes, and Patrick Bours. 2010. Improved gait recognition performance using cycle matching. In Proceedings of the IEEE 24th International Conference on Advanced Information Networking and Applications Workshops (WAINA'10). IEEE, 836-841. 
[36] Davrondzhon Gafurov, Einar Snekkenes, and Tor Erik Buvarp. 2006. Robustness of biometric gait authentication against impersonation attack. In Proceedings of the OTM Confederated International Conferences on the Move to Meaningful Internet Systems. Springer, 479-488.

[37] Shirley Gaw and Edward W. Felten. 2006. Password management strategies for online accounts. In Proceedings of the 2nd Symposium on Usable Privacy and Security. ACM, 44-55.

[38] Giacomo Giorgi, Fabio Martinelli, Andrea Saracino, and Mina Sheikhalishahi. 2017. Try walking in my shoes, if you can: Accurate gait recognition through deep learning. In Proceedings of the International Conference on Computer Safety, Reliability, and Security. Springer, 384-395.

[39] M. Günther, P. Hu, C. Herrmann, C. H. Chan, M. Jiang, S. Yang, A. R. Dhamija, D. Ramanan, J. Beyerer, J. Kittler, M. A. Jazaery, M. I. Nouyed, G. Guo, C. Stankiewicz, and T. E. Boult. 2017. Unconstrained face detection and open-set face recognition challenge. In Proceedings of the IEEE International foint Conference on Biometrics (IFCB'17). 697-706.

[40] Abdenour Hadid, Mohammad Ghahramani, Vili Kellokumpu, Matti Pietikäinen, John Bustard, and Mark Nixon. 2012. Can gait biometrics be spoofed? In Proceedings of the 21st International Conference on Pattern Recognition (ICPR'12). IEEE, 3280-3283.

[41] William J. Hardcastle and Nigel Hewlett. 2006. Coarticulation: Theory, Data and Techniques. Cambridge University Press.

[42] Jeffrey M. Hausdorff, Patrick L. Purdon, C. K. Peng, Z. V. I. Ladin, Jeanne Y. Wei, and Ary L. Goldberger. 1996. Fractal dynamics of human gait: Stability of long-range correlations in stride interval fluctuations. 7 . Appl. Physiol. 80, 5 (1996), 1448-1457.

[43] Eiji Hayashi and Jason Hong. 2011. A diary study of password usage in daily life. In Proceedings of the SIGCHI Conference on Human Factors in Computing Systems. ACM, 2627-2630.

[44] Seyed Amir Hoseini-Tabatabaei, Alexander Gluhak, and Rahim Tafazolli. 2013. A survey on smartphone-based systems for opportunistic user context recognition. ACM Comput. Surveys 45, 3 (2013), 27.

[45] Rob J. Hyndman. 2011. Moving averages. In International Encyclopedia of Statistical Science. Springer, 866-869.

[46] Ankita Jain and Vivek Kanhangad. 2018. Gender classification in smartphones using gait information. Expert Syst. Appl. 93 (2018), 257-266.

[47] Xianta Jiang, Kelvin H. T. Chu, Mahta Khoshnam, and Carlo Menon. 2018. A wearable gait phase detection system based on force myography techniques. Sensors 18, 4 (2018), 1279.

[48] Andrew H. Johnston and Gary M. Weiss. 2015. Smartwatch-based biometric gait recognition. In Proceedings of the IEEE 7th International Conference on Biometrics Theory, Applications and Systems (BTAS'15). IEEE, 1-6.

[49] Felix Juefei-Xu, Chandrasekhar Bhagavatula, Aaron Jaech, Unni Prasad, and Marios Savvides. 2012. Gait-id on the move: Pace independent human identification using cell phone accelerometer dynamics. In Proceedings of the IEEE 5th International Conference on Biometrics: Theory, Applications and Systems (BTAS'12). IEEE, 8-15.

[50] Joarder Kamruzzaman and Rezaul K. Begg. 2006. Support vector machines and other pattern recognition approaches to the diagnosis of cerebral palsy gait. IEEE Trans. Biomed. Eng. 53, 12 (2006), 2479-2490.

[51] Jochen Klucken, Jens Barth, Patrick Kugler, Johannes Schlachetzki, Thore Henze, Franz Marxreiter, Zacharias Kohl, Ralph Steidl, Joachim Hornegger, Bjoern Eskofier et al. 2013. Unbiased and mobile gait analysis detects motor impairment in Parkinson's disease. PloS One 8, 2 (2013), e56956.

[52] Jennifer R. Kwapisz, Gary M. Weiss, and Samuel A. Moore. 2010. Cell phone-based biometric identification. In Proceedings of the 4th IEEE International Conference on Biometrics: Theory Applications and Systems (BTAS'10). IEEE, $1-7$.

[53] Tracey K. M. Lee, Mohammed Belkhatir, and Saeid Sanei. 2014. A comprehensive review of past and present visionbased techniques for gait recognition. Multimedia Tools Appl. 72, 3 (2014), 2833-2869.

[54] Hong Lu, Jonathan Huang, Tanwistha Saha, and Lama Nachman. 2014. Unobtrusive gait verification for mobile phones. In Proceedings of the ACM International Symposium on Wearable Computers. ACM, 91-98.

[55] Richard G. Lyons. 2011. Understanding Digital Signal Processing, 3/E. Pearson Education India.

[56] Yasushi Makihara, Ngo Thanh Trung, Hajime Nagahara, Ryusuke Sagawa, Yasuhiro Mukaigawa, and Yasushi Yagi. 2010. Phase registration of a single quasi-periodic signal using self dynamic time warping. In Proceedings of the Asian Conference on Computer Vision. Springer, 667-678.

[57] Aakarsh Malhotra, Anush Sankaran, Apoorva Mittal, Mayank Vatsa, and Richa Singh. 2017. Fingerphoto authentication using smartphone camera captured under varying environmental conditions. In Human Recognition in Unconstrained Environments: Using Computer Vision, Pattern Recognition and Machine Learning Methods for Biometrics, Maria De Marsico, Michele Nappi, and Hugo Proença (Eds.). Academic Press, 119-144.

[58] Stéphane Mallat. 1999. A Wavelet Tour of Signal Processing. Academic Press.

[59] Jani Mäntyjärvi, Mikko Lindholm, Elena Vildjiounaite, Satu-Marja Mäkelä, and H. A. Ailisto. 2005. Identifying users of portable devices from gait pattern with accelerometers. In Proceedings of the IEEE International Conference on Acoustics, Speech, and Signal Processing (ICASSP'05), vol. 2. IEEE, ii-973. 
[60] Alessio Mecca. 2018. Impact of gait stabilization: A study on how to exploit it for user recognition. In Proceedings of the 14th International Conference on Signal-Image Technology \& Internet-Based Systems (SITIS'18). IEEE, 553-560.

[61] Pallavi Meharia and Dharma P. Agrawal. 2015. The human key: Identification and authentication in wearable devices using gait. f. Info. Privacy Security 11, 2 (2015), 80-96.

[62] Lee Middleton, Alex Buss, Alex Bazin, Mark S. Nixon et al. 2005. A floor sensor system for gait recognition. In Proceedings of the 4th IEEE Workshop on Automatic Identification Advanced Technologies. IEEE, 171-176.

[63] Muhammad Muaaz and René Mayrhofer. 2017. Smartphone-based gait recognition: from authentication to imitation. IEEE Trans. Mobile Comput. 16, 11 (2017), 3209-3221.

[64] Alvaro Muro-De-La-Herran, Begonya Garcia-Zapirain, and Amaia Mendez-Zorrilla. 2014. Gait analysis methods: An overview of wearable and non-wearable systems, highlighting clinical applications. Sensors 14, 2 (2014), 3362-3394.

[65] Lewis M. Nashner and Gin McCollum. 1985. The organization of human postural movements: A formal basis and experimental synthesis. Behav. Brain Sci. 8, 1 (1985), 135-150.

[66] Thanh Trung Ngo, Yasushi Makihara, Hajime Nagahara, Yasuhiro Mukaigawa, and Yasushi Yagi. 2014. The largest inertial sensor-based gait database and performance evaluation of gait-based personal authentication. Pattern Recogn. 47, 1 (2014), 228-237.

[67] Trung Thanh Ngo, Yasushi Makihara, Hajime Nagahara, Yasuhiro Mukaigawa, and Yasushi Yagi. 2014. Orientationcompensative signal registration for owner authentication using an accelerometer. IEICE Trans. Info. Syst. 97, 3 (2014), 541-553.

[68] Claudia Nickel, Holger Brandt, and Christoph Busch. 2011. Classification of acceleration data for biometric gait recognition on mobile devices.BIOSIG 11 (2011), 57-66.

[69] Claudia Nickel, Christoph Busch, Sathyanarayanan Rangarajan, and Manuel Möbius. 2011. Using hidden markov models for accelerometer-based biometric gait recognition. In Proceedings of the IEEE 7th International Colloquium on Signal Processing and its Applications (CSPA'11). IEEE, 58-63.

[70] Claudia Nickel, Mohammad O. Derawi, Patrick Bours, and Christoph Busch. 2011. Scenario test of accelerometerbased biometric gait recognition. In Proceedings of the 3rd International Workshop on Security and Communication Networks (IWSCN'11). IEEE, 15-21.

[71] Claudia Nickel, Tobias Wirtl, and Christoph Busch. 2012. Authentication of smartphone users based on the way they walk using k-NN algorithm. In Proceedings of the 8th International Conference on Intelligent Information Hiding and Multimedia Signal Processing (IIH-MSP'12). IEEE, 16-20.

[72] Michael Fitzgerald Nowlan. 2009. Human identification via gait recognition using accelerometer gyro forces. Yale Computer Science. Retrieved from http://www.cs.yale.edu/homes/mfn3/pub/mfn_gait_id.pdf.

[73] Robert J. Orr and Gregory D. Abowd. 2000. The smart floor: A mechanism for natural user identification and tracking. In CHI'00 Extended Abstracts on Human Factors in Computing Systems. ACM, 275-276.

[74] Gang Pan, Ye Zhang, and Zhisheng Wu. 2009. Accelerometer-based gait recognition via voting by signature points. Electron. Lett. 45, 22 (2009), 1116-1118.

[75] Todd C. Pataky, Tingting Mu, Kerstin Bosch, Dieter Rosenbaum, and John Y. Goulermas. 2012. Gait recognition: Highly unique dynamic plantar pressure patterns among 104 individuals. f. Roy. Soc. Interface 9, 69 (2012), 790-800.

[76] Antonio Pedotti. 1977. A study of motor coordination and neuromuscular activities in human locomotion. Biol. Cybernet. 26, 1 (1977), 53-62.

[77] A. Pedotti, V. V. Krishnan, and L. Stark. 1978. Optimization of muscle-force sequencing in human locomotion. Math. Biosci. 38, 1-2 (1978), 57-76.

[78] Ronald Poppe. 2010. A survey on vision-based human action recognition. Image Vision Comput. 28, 6 (2010), 976-990.

[79] T. Pozzo, A. Berthoz, and L. Lefort. 1990. Head stabilization during various locomotor tasks in humans. Exper. Brain Res. 82, 1 (1990), 97-106.

[80] Chandra Prakash, Rajesh Kumar, and Namita Mittal. 2018. Recent developments in human gait research: Parameters, approaches, applications, machine learning techniques, datasets and challenges. Artific. Intell. Rev. 49, 1 (2018), 1-40.

[81] Anshul Rai, Krishna Kant Chintalapudi, Venkata N. Padmanabhan, and Rijurekha Sen. 2012. Zee: Zero-effort crowdsourcing for indoor localization. In Proceedings of the 18th Annual International Conference on Mobile Computing and Networking. ACM, 293-304.

[82] V. Joseph Raj and S. Balamurugan. 2017. Survey of current trends in human gait recognition approaches. Int. F. Adv Res. 5 (10 2017), 1851-1864.

[83] H. J. Ralston, V. Inman, and F. Todd. 1981. Human Walking. Williams and Wilkins, Baltimore.

[84] Daniele Riboni and Claudio Bettini. 2009. Context-aware activity recognition through a combination of ontological and statistical reasoning. In Proceedings of the International Conference on Ubiquitous Intelligence and Computing. Springer, 39-53.

[85] Liu Rong, Zhou Jianzhong, Liu Ming, and Hou Xiangfeng. 2007. A wearable acceleration sensor system for gait recognition. In Proceedings of the 2nd IEEE Conference on Industrial Electronics and Applications. IEEE, 2654-2659. 
[86] Liu Rong, Duan Zhiguo, Zhou Jianzhong, and Liu Ming. 2007. Identification of individual walking patterns using gait acceleration. In Proceedings of the 1st International Conference on Bioinformatics and Biomedical Engineering. IEEE, 543-546.

[87] Yiran Shen, Wen Hu, Mingrui Yang, Bo Wei, Simon Lucey, and Chun Tung Chou. 2014. Face recognition on smartphones via optimised sparse representation classification. In Proceedings of the 13th International Symposium on Information Processing in Sensor Networks. IEEE Press, 237-248.

[88] Sebastijan Sprager and Matjaz B. Juric. 2015. Inertial sensor-based gait recognition: A review. Sensors 15, 9 (2015), 22089-22127.

[89] Jaakko Suutala and Juha Röning. 2004. Towards the adaptive identification of walkers: Automated feature selection of footsteps using distinction sensitive LVQ. In Proceedings of the International Workshop on Processing Sensory Information for Proactive Systems (PSIPS’04). 14-15.

[90] Takahiro Takeda, Kazuhiko Taniguchi, Kazunari Asari, Kei Kuramoto, Syoji Kobashi, and Yutaka Hata. 2009. Biometric personal authentication by one step foot pressure distribution change by load distribution sensor. In Proceedings of the IEEE International Conference on Fuzzy Systems (FUZZ-IEEE'09). IEEE, 906-910.

[91] Thiago Teixeira, Deokwoo Jung, Gershon Dublon, and Andreas Savvides. 2009. PEM-ID: Identifying people by gaitmatching using cameras and wearable accelerometers. In Proceedings of the 3rd ACM/IEEE International Conference on Distributed Smart Cameras (ICDSC'09). IEEE, 1-8.

[92] Ngo Thanh Trung, Yasushi Makihara, Hajime Nagahara, Ryusuke Sagawa, Yasuhiro Mukaigawa, and Yasushi Yagi. 2011. Phase registration in a gallery improving gait authentication. In Proceedings of the International foint Conference on Biometrics (IFCB'11). IEEE, 1-7.

[93] Pavan Turaga, Rama Chellappa, Venkatramana S. Subrahmanian, and Octavian Udrea. 2008. Machine recognition of human activities: A survey. IEEE Trans. Circ. Syst. Video Technol. 18, 11 (2008), 1473-1488.

[94] Christopher L. Vaughan, Brian L. Davis, C. O. Jeremy et al. 1999. Dynamics of human gait. Human Kinetics Publishers.

[95] Elena Vildjiounaite, Satu-Marja Mäkelä, Mikko Lindholm, Reima Riihimäki, Vesa Kyllönen, Jani Mäntyjärvi, and Heikki Ailisto. 2006. Unobtrusive multimodal biometrics for ensuring privacy and information security with personal devices. In Proceedings of the International Conference on Pervasive Computing. Springer, 187-201.

[96] Dayong Wang, Charles Otto, and Anil K. Jain. 2017. Face search at scale. IEEE Trans. Pattern Anal. Mach. Intell. 39, 6 (2017), 1122-1136.

[97] Gary M. Weiss and Haym Hirsh. 1998. Learning to predict rare events in event sequences. In Proceedings of the 4th International Conference on Knowledge Discovery and Data Mining. 359-363.

[98] Weitao Xu, Yiran Shen, Yongtuo Zhang, Neil Bergmann, and Wen Hu. 2017. Gait-watch: A context-aware authentication system for smart watch based on gait recognition. In Proceedings of the 2nd International Conference on Internet-of-Things Design and Implementation. ACM, 59-70.

[99] Zhaojun Xue, Dong Ming, Wei Song, Baikun Wan, and Shijiu Jin. 2010. Infrared gait recognition based on wavelet transform and support vector machine. Pattern Recogn. 43, 8 (2010), 2904-2910.

[100] Kuo-Hui Yeh, Chunhua Su, Wayne Chiu, and Lu Zhou. 2018. I walk, therefore I am: Continuous user authentication with plantar biometrics. IEEE Commun. Mag. 56, 2 (2018), 150-157.

[101] Yuting Zhang, Gang Pan, Kui Jia, Minlong Lu, Yueming Wang, and Zhaohui Wu. 2015. Accelerometer-based gait recognition by sparse representation of signature points with clusters. IEEE Trans. Cybernet. 45, 9 (2015), 1864-1875.

[102] Yu Zhong and Yunbin Deng. 2014. Sensor orientation invariant mobile gait biometrics. In Proceedings of the IEEE International foint Conference on Biometrics (IFCB'14). IEEE, 1-8.

[103] Qin Zou, Lihao Ni, Qian Wang, Qingquan Li, and Song Wang. 2018. Robust gait recognition by integrating inertial and RGBD sensors. IEEE Trans. Cybernet. 48, 4 (2018), 1136-1150.

Received June 2018; revised February 2019; accepted June 2019 